\title{
Secure Compilation to Protected Module Architectures
}

\author{
Marco Patrignani and Pieter Agten and Raoul Strackx and Bart Jacobs, iMinds-DistriNet, \\ Dept. Computer Science, KU Leuven, Belgium \\ and Dave Clarke, Dept. Information Technology, Uppsala, Sweden and iMinds-DistriNet, Dept. \\ Computer Science, KU Leuven, Belgium \\ and Frank Piessens, iMinds-DistriNet, Dept. Computer Science, KU Leuven, Belgium
}

\begin{abstract}
A fully abstract compiler prevents security features of the source language from being bypassed by an attacker operating at the target language level. Unfortunately, developing fully abstract compilers is very complex, and it is even more so when the target language is an untyped assembly language. To provide a fully abstract compiler that targets untyped assembly, it has been suggested to extend the target language with a protected module architecture — an assembly-level isolation mechanism which can be found in nextgeneration processors. This paper provides a fully abstract compilation scheme whose source language is an object-oriented, high-level language and whose target language is such an extended assembly language. The source language enjoys features such as dynamic memory allocation and exceptions. Secure compilation of first-order method references, cross-package inheritance and inner classes is also presented. Moreover, this paper contains the formal proof of full abstraction of the compilation scheme. Measurements of the overhead introduced by the compilation scheme indicate that it is negligible.
\end{abstract}

Categories and Subject Descriptors: D.2.3 [Coding Tools and Techniques]: Object-oriented programming; D.2.4 [Software/Program Verification]: Formal Methods; D.3.4 [Processors]: Compilers; D.4.6 [Security and Protection]: Verification

General Terms: Secure compilation, object-oriented programming, untyped machine code, security

Additional Key Words and Phrases: Fully abstract compilation, protected module architecture

ACM Reference Format:

Marco Patrignani, Pieter Agten, Raoul Strackx, Bart Jacobs, Dave Clarke and Frank Piessens, 2014. Secure compilation to Protected Module Architectures. ACM Trans. Program. Lang. Syst. ?, ?, Article ?? (January ????), 51 pages.

DOI : http://dx.doi.org/10.1145/0000000.0000000

\section{INTRODUCTION}

Modern high-level languages such as ML, Java or Scala offer security features to programmers in the form of type systems, module systems, encapsulation primitives and so forth. In order for these high-level programs to be executed, they are input to other programs: compilers (in certain cases they are given to interpreters which are even-

This work has been supported in part by the Intel Lab's University Research Office. This research is also partially funded by the Research Fund KU Leuven, and by the EU FP7 project NESSoS. With the financial support from the Prevention of and Fight against Crime Programme of the European Union (B-CCENTRE). Marco Patrignani and Pieter Agten hold a Ph.D. fellowship from the Research Foundation Flanders (FWO). Raoul Strackx is a Ph.D. fellow of the agency for Innovation by Science and Technology (IWT).

Author's addresses: Marco Patrignani, Pieter Agten, Raoul Strackx, Bart Jacobs and Frank Piessens, Computer Science Department, KU Leuven, Belgium, email: \{first.last\}@cs.kuleuven.be. Dave Clarke (Current address) Department of Information Technology, Uppsala, Sweden, email: \{first.last\}@it.uu.se.

Permission to make digital or hard copies of part or all of this work for personal or classroom use is granted without fee provided that copies are not made or distributed for profit or commercial advantage and that copies show this notice on the first page or initial screen of a display along with the full citation. Copyrights for components of this work owned by others than ACM must be honored. Abstracting with credit is permitted. To copy otherwise, to republish, to post on servers, to redistribute to lists, or to use any component of this work in other works requires prior specific permission and/or a fee. Permissions may be requested from Publications Dept., ACM, Inc., 2 Penn Plaza, Suite 701, New York, NY 10121-0701 USA, fax +1 (212) 869-0481, or permissions@acm.org.

(c) ???? ACM 0164-0925/????/01-ART?? \$15.00

DOI : http://dx.doi.org/10.1145/0000000.0000000 
tually compiled). Compilers take programs written in a language, called the source language, and output other programs written (often) in a different language, called the target language. The target language is (often) machine code and it can be executed directly by the processor. Unfortunately, most target languages do not offer the same security features as high-level source languages. Consider assembly as the target language of a compiler. An attacker acting at the assembly level can inspect and modify the whole memory space, breaking security properties such as confidentiality and integrity of compiled code. One way to withstand such an attacker is by means of secure compilation, which makes a compiler produce target-level output as secure as its source-level input.

A way to achieve secure compilation is through a fully abstract compiler. A fully abstract compiler preserves (and reflects) all abstractions of the source language in the target language, including security abstractions. So, when the target of a fully abstract compiler is assembly, the power of a low-level attacker is reduced to that of a source-level attacker.

Informally, a compiler is fully abstract when it translates indistinguishable sourcelevel programs into indistinguishable target-level programs. Formally, a fully abstract compiler preserves and reflects contextual equivalence between source- and targetlevel programs. Two programs are contextually equivalent if they cannot be distinguished by a third one. For example, consider two instances $P_{1}$ and $P_{2}$ of the same program which contain two different values in the same variable $v$. Denote their fully abstract-compiled counterparts by $P_{1}^{\downarrow}$ and $P_{2}^{\downarrow}$. If $P_{1}$ and $P_{2}$ are contextually equivalent, then $P_{1}^{\downarrow}$ and $P_{2}^{\downarrow}$ must also be. So, if the content of $v$ is confidential (for example, the value stored in $v$ could be private and never communicated), no program interacting with $P_{1}^{\downarrow}$ or $P_{2}^{\downarrow}$ can discern it. Notice that a fully abstract compiler does not eliminate source-level security flaws. It is, in a sense, conservative, as it introduces no more vulnerabilities at the target-level than the ones already exploitable at the source level.

The notion of fully abstract compilation also has consequences for code reasoning, as it allows source-level reasoning. When writing code, programmers need to consider both the source-level setting where the code is written and the target-level setting where the code runs. Source-level reasoning means that a programmer needs only to reason about the behaviour of source-level code. The target-level code is guaranteed to behave as its source-level counterpart even in the presence of target-level attacks.

With these premises, it is clear that fully abstract compilation is hard to achieve. Compilation of Java to JVM or of $\mathrm{C}^{\#}$ to the .NET framework are some of the examples where compilation is not fully abstract, as Kennedy presented [Kennedy 2006]. Nevertheless, techniques to achieve fully abstract compilation exist. They rely on address space layout randomisation [Abadi and Plotkin 2012; Jagadeesan et al. 2011], typebased invariants [Ahmed and Blume 2011; Fournet et al. 2013], and enhancing the target language with a protected module architecture [Agten et al. 2012; Patrignani et al. 2013].

This paper describes a fully abstract compilation scheme from a secure high-level language to untyped assembly language enhanced with a protected module architecture. Protected module architectures offer a fine-grained, program counter-based memory access control mechanism which provides isolation for specific memory areas. This isolation mechanism is adopted to preserve source-level security properties in the output of the compiler. The target language is similar to a modern processor architecture, so the compilation scheme handles subtleties such as flags and registers. The source language is a component-based, java-like object-oriented programming language that borrows extensively from the Java Jr. language of Jeffrey and Rathke [Jeffrey and Rathke 2005b], extending it with exceptions. 
The contributions of this paper are the following:

- the presentation of a fully abstract compilation scheme for a strongly-typed, component-based, object-oriented programming language to untyped assembly language;

— the highlighting of mistakes that make a naïve compilation scheme not fully abstract and their correction;

- the formalisation of both the source and the target languages and the proof of full abstraction of the compilation scheme;

- the benchmarking of the overhead introduced by the fully abstract compiler.

The prototype of the compiler described in this paper was implemented by means of additional LLVM passes [Lattner and Adve 2004].

The results of this paper are expressed w.r.t. a specific target language adopting a specific protected module architecture. The lessons learned can be applied to target languages adopting different architectures up to minor technical changes. In fact, the paper highlights what are the features that the protected module architectures needs to implement to provide the isolation mechanism necessary for the compilation scheme to be fully abstract.

The paper firstly presents an informal overview of background notions such as the source and target languages (Section 2), then it describes the secure compilation scheme (Section 3). The paper then formalises the source and target languages (Section 4) and provides the formal proof of full abstraction of the compilation scheme (Section 5). Then, the paper presents the benchmarking of the overhead introduced by the secure compilation scheme (Section 6), followed by a discussion on its extensions and limitations (Section 7). Finally, the paper discusses related work (Section 8), future work and concludes (Section 9).

\section{INFORMAL OVERVIEW}

This section firstly presents a comparison between different target-level protection mechanisms (Section 2.1), then it informally introduces both the target and the source languages of the fully abstract compiler (Section 2.2 and 2.3). This section then gives an overview of how we will carry out the proof of full abstraction of the compilation scheme of Section 3 (Section 2.4). Contextual equivalence is then presented as a good candidate for security policies enforcement through compilation (Section 2.5). Finally, this section present the threat model for this paper (Section 2.6).

\subsection{Low-level Protection Mechanism}

In order to safeguard low-level assembly programs from malicious attackers, a number of alternatives arise. In the following we review the three most interesting ones: address space layout randomisation, protected module architectures and processes; a review of additional ones is left for Section 8. We do not give a thorough review of all existing low-level protection mechanism, the interested reader is referred to the $\mathrm{PhD}$. thesis of Yves Younan for an in-depth analysis of the subject [Younan 2008]. Alternatives to using low-level protection mechanism include using massive-scale, diversified software [Homescu et al. 2013; Larsen et al. 2014]

Address Space Layout Randomisation: ASLR. ASLR is a technique that randomises the memory layout of key data areas of a program such as the base of the stack, of the heap, of libraries, etc. The executable is divided in segments whose order is randomised by the dynamic linker (i.e. just before running the executable). This technique is used to hinder an attacker from mounting "return to libc" attacks, or from using previously acquired knowledge of the location of certain data to access that data. 
ASLR has been used to achieve (probabilistic) fully abstract compilation of the simply-typed $\lambda$-calculus [Abadi and Plotkin 2012] and of the same language extended with dynamic memory allocation, first and higher-order references and call/cc [Jagadeesan et al. 2011]. It requires a small trusted computing base (TCB) consisting of the randomisation function used to generate the different memory layouts.

The major drawback of ASLR (and of related techniques such as stirring [Wartell et al. 2012]) for secure compilation is that it relies on a good randomisation function, so it only provides probabilistic guarantees. Moreover, there have been successful derandomisation attacks to existing ASLR implementations [Shacham et al. 2004] which could be used to violate the security of a compiler relying on ASLR. Finally, certain attacks, such as the ones presented in Problem 5 in Section 3, focus on corrupting data instead of the control flow of execution. These are not simply solved with ASLR and require additional care, as discussed in Section 3.

Protected Module Architectures: PMA. PMA provides assembly code with the ability to create protected modules. Like their high-level counterpart, such as ML modules, these modules offer an interface mechanism to allow interoperation with code that is not in the module. Additionally, these modules isolate what is placed within the module boundaries.

The most common way to implement PMA is through program counter-based memory access control mechanisms [McCune et al. 2010; McCune et al. 2008; Noorman et al. 2013; Singaravelu et al. 2006; Strackx and Piessens 2012; McKeen et al. 2013]. We review these mechanisms from the work of Patrignani et al. [Patrignani et al. 2013] and Strackx and Piessens [Strackx and Piessens 2012]. This mechanism logically divides the memory into a protected and an unprotected section. The protected section is further divided into a code and a data section. The code section contains a variable number of entry points: the only protected addresses to which instructions in unprotected memory can jump and execute. The data section is accessible only from the protected section. The size and location of each memory section are specified in a memory descriptor. The table below summarises the access control model enforced by the protection mechanism.

\begin{tabular}{|c|c|c|c|c|}
\hline From $\backslash$ To & \multicolumn{3}{|c|}{ Protected } & Unprotected \\
& Entry Point & Code & Data & \\
\hline Protected & r x & r x & r w & r w x \\
\hline Unprotected & $\mathbf{x}$ & & & r w x \\
\hline
\end{tabular}

This protection mechanism provides a secure environment for code that needs to be protected from a potentially malicious surrounding environment. It is appealing in the context of embedded systems, where kernel-level protection mechanisms are often lacking. Very recently, Intel publicly announced Software Guard Extensions (Intel SGX), a hardware implementation of a Protected Module Architecture. Hence PMA support will be broadly available in mainstream processors within a few years, and any processor with PMA support can benefit from the secure compilation techniques proposed in this paper. Moreover, secure compilation and PMA aid the verification of properties of $\mathrm{C}$ code placed inside a protected module [Agten et al. 2015]. Finally, the TCB of PMA can be very small [McCune et al. 2008; Singaravelu et al. 2006; Strackx and Piessens 2012; Strackx et al. 2010] or even zero-software [Eldefrawy et al. 2012; Noorman et al. 2013; McKeen et al. 2013]. The interested reader is referred to the work of Strackx et al. [Strackx et al. 2013] for further inquiries on this line of security research. 
Processes. The same access control policy of PMA can be implemented by using operating system-level processes. In this setting, each process gets a different address space, which provides a protected memory where other processes have no access rights. Different processes can communicate via message passing, effectively calling functions implemented in each other's address space. Additionally, shared memory can be used for larger data management.

There are two drawbacks for this approach. Firstly, processes and inter-process communication are relatively heavyweight constructs to use. Crossing the boundary between one process and another is more costly than switching between protected and unprotected code (and vice-versa) in current PMA implementations. Additionally, the TCB of this protection mechanism includes the whole operating system, which is much larger than current PMA implementations.

The reduced TCB, the security benefits and the novelty in choosing it as a protection mechanism motivate the choice of PMA to protect the target language of the compilation scheme.

\subsection{The Target Language $\mathcal{A}+\mathrm{I}$, Informally}

To model a realistic compilation scheme, the target language should be close to what is used by modern processors. For this reason this paper adopts $\mathcal{A}+l$ (acronym of Assembly plus Isolation), a low-level language that models a standard Von Neumann machine consisting of a program counter, a registers file, a flags register and memory space [Agten et al. 2012; Patrignani et al. 2013; Patrignani and Clarke 2014], enhanced with a protected module architecture. The registers file contains twelve general-purpose registers $r_{0}-r_{11}$ and a stack pointer register SP, which points to the top of the call stack. The flags register contains two flags: a zero flag ZF and a sign flag SF which are set when arithmetic operations output a zero or a negative number, respectively. The call stack is located in unprotected memory. Instructions executed by the language are listed in Fig. 1. For the sake of simplicity, assume the architecture targeted by the language works with $\ell$ bit-long words, where $\ell$ is a power of 2 . This allows the formalisation presented to scale to architectures with words of different sizes. A complete formalisation of $\mathcal{A}+\mathrm{l}$ is delayed until Section 4.1. The protection mechanism

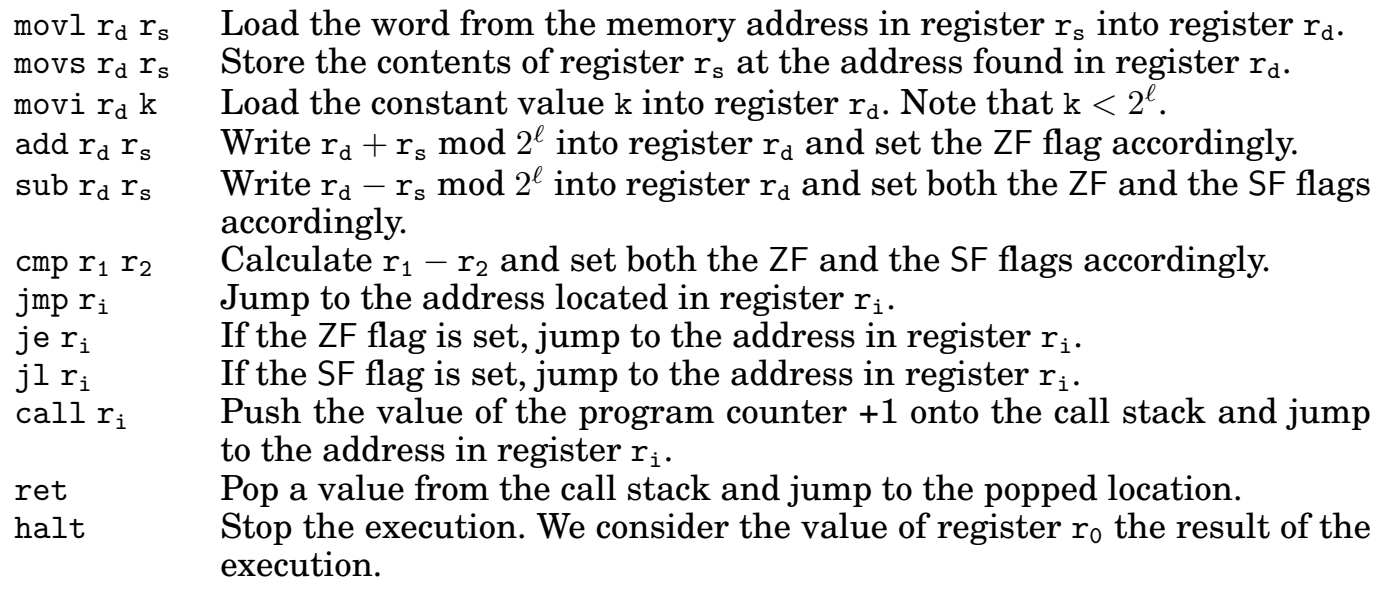

Fig. 1: Instruction set $\mathcal{I}$ of language $\mathcal{A}+$ l.

affects the semantics of the language, preventing the execution of certain instructions, 
in accordance with the PMA access control policy presented in Section 2.1. Following are some code snippets that exemplify the semantics of $\mathcal{A}+$ I. In all examples concerning $\mathcal{A}+\mathrm{l}$ code, assume the presence of a protected memory section spanning from address 100 to 200 , with a single entry point at address 100 . Let $P_{s}$ denote the code located in the protected section and $P_{u}$ denote the code located in the unprotected one. Every instruction is preceded by the address where it is located; execution starts at address 0 .

Example 2.1 (No execution of code in the protected memory partition). $P_{u} \quad$ initialises register $r_{0}$ to 101 (line 1) and then jumps to that address (line 2).

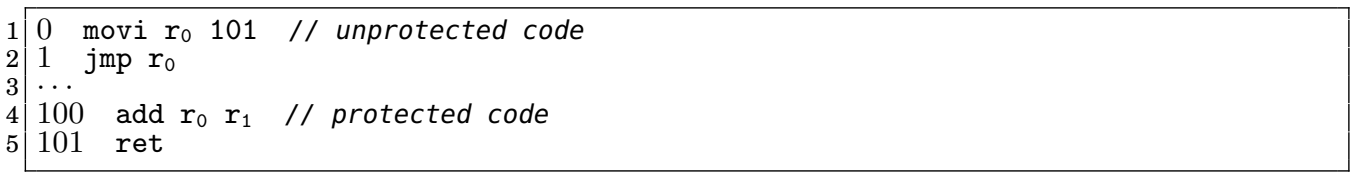

Since address 101 is not an entry point of the protected memory section, the jump of $P_{u}$ does not succeed and execution is halted (this can happen in a variety of ways, for example, in our prototype detailed in Section 6.1, the execution is suspended and trapped by the hypervisor [Strackx and Piessens 2012]).

Example 2.2 (No reading / writing the protected code section). $P_{u}$ initialises register $r_{0}$ to 101 (line 1) and register $r_{1}$ to 20 (line 2), then it writes the content of $r_{1}$ at the address in $r_{0}$ (line 3 ).

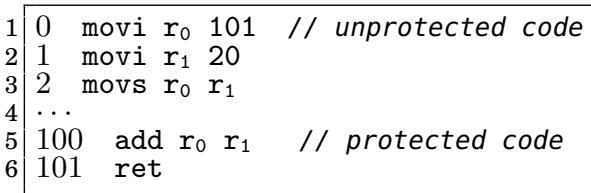

Since address 101 is protected, $P_{u}$ cannot write there, so execution is halted, as in Example 2.1. Analogously, if the instruction of line 2 were replaced with movl $r_{0} r_{1}$, the execution fails. In that case, $P_{u}$ would be attempting to read the protected memory section, while it does not have that privilege.

Example 2.3 (Interoperation between protected and unprotected code). $P_{u} \quad$ initialises register $r_{0}$ to 12 (line 1), register $r_{1}$ to 10 (line 2), register $r_{5}$ to 100 (line 3) and then calls to the protected function located at address 100 (line 4), storing address 4 on the call stack. $P_{s}$ subtracts registers $r_{0}$ and $r_{1}$ (line 6) and, if the result is greater than or equal to zero, it returns that result (line 9). Otherwise, if the result is less than zero, $P_{s}$ jumps to address 104 (lines 7,8), and returns 0 (lines 10,11). Execution then continues in unprotected memory at address 4 (line 5, omitted), which is the address popped from the call stack.

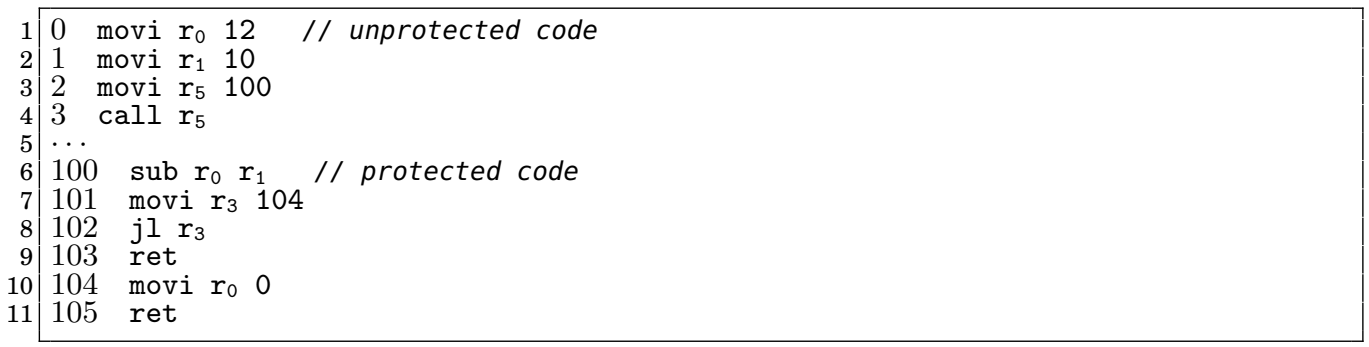




\subsection{The High-level Language $\mathcal{J}+\mathrm{E}$, Informally}

The high-level language adopted by the compilation scheme is $\mathcal{J}+\mathrm{E}$ (acronym of $\mathcal{J}$ ava plus Encapsulation): a strongly-typed, single-threaded, component-based, objectoriented language that enforces private fields and public methods. $\mathcal{J}+\mathrm{E}$ extends the Java Jr. language of Jeffrey and Rathke [Jeffrey and Rathke 2005b] with local variables and exceptions. It was chosen since it provides a clear notion of encapsulation for a high-level component, which makes for simpler reasoning about the secure compilation scheme.

$\mathcal{J}+\mathrm{E}$ supports many of the basic constructs one expects from a programming language (Fig. 2). A program in $\mathcal{J}+\mathrm{E}$ is called a component, it is a collection of sealed

\begin{tabular}{|c|c|}
\hline components & $C::=\bar{P}$ \\
\hline packages & $P::=\left\{\right.$ package $\left.p ; \overline{D_{i}}\right\} \mid\left\{\right.$ package $\left.p ; \overline{D_{e}}\right\}$ \\
\hline import declarations & $\begin{aligned} D_{i}::= & \text { interface } i \text { extends } \bar{t}\left\{\overline{M_{t}}\right\} \\
& \mid \text { extern } o: t\end{aligned}$ \\
\hline export declarations & $\begin{aligned} D_{e}::= & \text { class } c \text { extends } t \text { implements } \bar{t}\left\{K \overline{F_{t}} \bar{M}\right\} \\
& \mid \text { object } o: t \text { implements } \bar{t}\{\bar{F}\}\end{aligned}$ \\
\hline constructors & $K::=c\left(\bar{f}: \bar{t}, \overline{f^{\prime}}: \overline{t^{\prime}}\right)\left\{\operatorname{super}(\bar{f}) ;\right.$ this. $\left.\overline{f^{\prime \prime}}=\overline{f^{\prime}}\right\}$ \\
\hline fields & $F::=$ private $f=v$ \\
\hline field types & $F_{t}::=$ private $f: t$ \\
\hline methods & $M::=$ public $m(\bar{x}: \bar{t}): t$ [throws $t]\{$ return $E ;\}$ \\
\hline method types & $M_{t}::=$ public $m(\bar{x}: \bar{t}): t$ [throws $\left.t\right]$ \\
\hline expressions & $\begin{aligned} E::= & v|x| E . f|E . f=E| E \cdot m(\bar{E}) \mid E \text { op } E \mid \text { exit } E \\
& |E ; E| E \text { in } p|\operatorname{var} x: t=E| \text { if }(E)\{E\} \text { else }\{E\} \\
& \mid \text { new } t(\bar{E})|\operatorname{try}\{E\} \operatorname{catch}(x: t)\{E\}| \text { throw } E\end{aligned}$ \\
\hline $\begin{array}{l}\text { types } \\
\text { operations } \\
\text { values }\end{array}$ & $\begin{aligned} t & :=p . c|p . i| p . c \text { in } p \mid p . c \text { in } *|\mathbf{O b j}| \text { Unit } \mid \text { Bool } \mid \text { Int } \\
\text { op } & :=+|-| \cdot|/| \wedge|\vee| \cdots \\
v & :=p . o \mid \text { unit } \mid \text { true } \mid \text { false }|n| \text { throw } v\end{aligned}$ \\
\hline
\end{tabular}

Fig. 2: Syntax of $\mathcal{J}+\mathrm{E}$. Denote a sequence of elements $E_{1}, \ldots, E_{n}$ with $\bar{E}$.

packages that communicate via interfaces and public objects. $\mathcal{J}+\mathrm{E}$ partitions packages into import and export ones. Import packages are analogous to the $\mathrm{h}$ header file of a $\mathrm{C}$ program. ${ }^{1}$ They define interfaces, which are named collections of method signatures, and externs, which are references to externally defined objects. Export packages provide an implementation of an import package. They define classes, which are named collections of method implementations and fields (also known as instance variables), and objects, which implement classes and provide bindings from fields to values. Methods in $\mathcal{J}+E$ can (optionally) throw exceptions. The top of the class hierarchy is Obj, the only primitive types in $\mathcal{J}+\mathrm{E}$ are Unit, inhabited by unit; Bool, inhabited by true and false; and Int, inhabited by word-sized integers. The expression $E$ in $p$ is borrowed

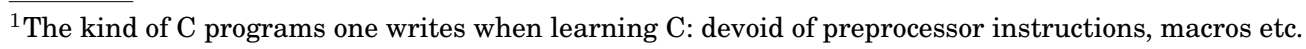


from Java Jr. It is a type coercion that allows the following: if the expression $E$ is welltyped to run in package $p$ with return type $t$, then the expression $E$ in $p$ is well-typed to run in any package $q$ with return type $t$, as long as $t$ is a visible type in $q$.

Listing 1 illustrates the package system of $\mathcal{J}+\mathrm{E}$. There, and in future code examples, we will massage the syntax of the presented examples for the sake of readability. It

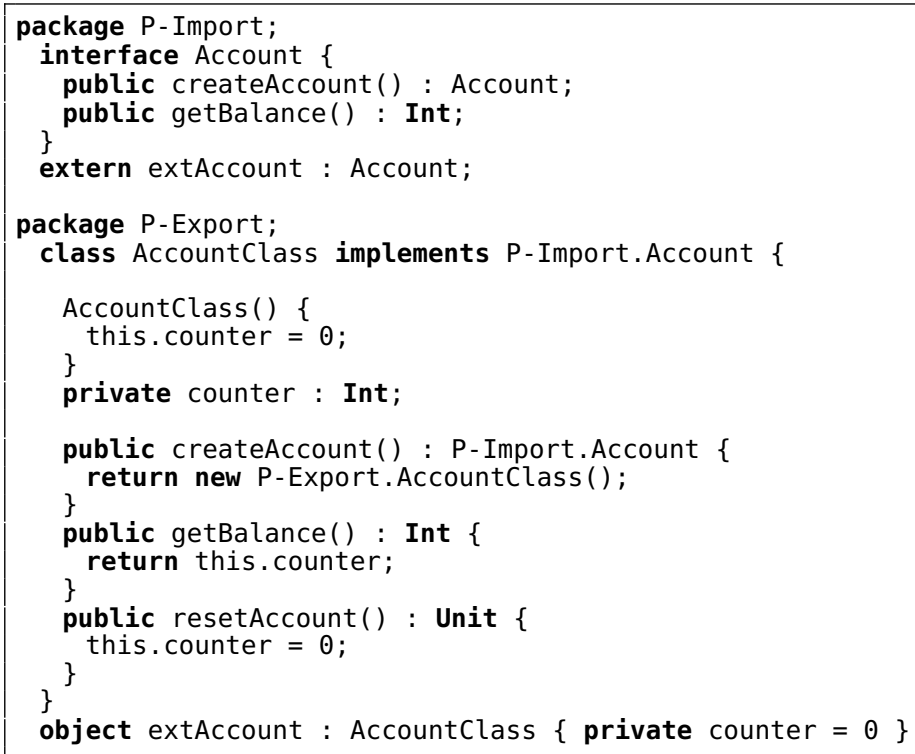

Listing 1: Example of the package system of $\mathcal{J}+\mathrm{E}$

contains two package declarations: $\mathrm{P}$-Import is an import package and P-Export is an export package implementing P-Import. P-Export provides class AccountClass that implements interface Account defined in P-Import. Object extAccount allocated in P-Export provides an implementation for the extern with the same name defined in P-Import.

One of the security mechanisms of $\mathcal{J}+\mathrm{E}$ is given by private fields, which can be used to define security properties such as confidentiality and integrity, as they are not accessible from outside the class declaring them. In $\mathcal{J}+\mathrm{E}$, classes are private to the package that contains their declarations. Objects are allocated in the same package as the class they instantiate. Due to this package system, for a package to be compiled it only needs the import packages of any package it depends on. As a result, formal parameters in methods have interface types, since classes that implement those interfaces are unknown. This discipline is called: programming to an interface. Additionally, since constructors are not exposed in interfaces, cross-package object allocation happens through factory methods. For example, the name of class Accountclass from Listing 1 is not visible from outside package $P$-Export, thus expressions of the form new P-Export.AccountClass () cannot be written outside P-Export.

\subsection{The Proof of Full Abstraction of the Compilation Scheme, Informally}

A fully abstract compilation scheme preserves and reflects contextual equivalence of source- and target-level programs. Informally speaking, two programs $C_{1}$ and $C_{2}$ are contextually equivalent if they behave the same for all possible programs they interact with. The programs that $C_{1}$ and $C_{2}$ can interact with are called contexts, they are 
(partial) programs with a hole. Once the hole is filled with either $C_{1}$ or $C_{2}$, the program is whole. For any context, if the behaviour of the whole program does not change when the hole is filled with either $C_{1}$ or $C_{2}$, then $C_{1}$ and $C_{2}$ are contextually equivalent [Plotkin 1977]. This notion is denoted as $C_{1} \simeq C_{2}$.

Given a source-level program $C_{1}$, denote the target level program that is produced by compiling it as $C_{1}^{\downarrow}$. Fully abstract compilation is formally expressed as: $C_{1} \simeq C_{2} \Longleftrightarrow$ $C_{1}^{\downarrow} \simeq C_{2}^{\downarrow}$. In order to prove this statement, the equivalence is split in two cases.

- The direction $C_{1}^{\downarrow} \simeq C_{2}^{\downarrow} \Rightarrow C_{1} \simeq C_{2}$ states that the compiler outputs target-level programs that behave as the corresponding source programs. This is what most compilers achieve, at times even certifying the result [Chlipala 2007; Leroy 2009]; we are not interested in this direction. This is thus assumed, the implications of this assumption are made explicit in Section 5.2.

- The direction $C_{1} \simeq C_{2} \Rightarrow C_{1}^{\downarrow} \simeq C_{2}^{\downarrow}$ states that source-level abstractions are preserved through compilation to the target level. Proving this direction requires reasoning about contexts, which is notoriously difficult [Ahmed and Blume 2011]. This is even more so in this setting, where contexts are low-level memories lacking any inductive structure. To avoid working with contexts, we replace the notion of contextual equivalence $(\simeq)$ at the target level, with that of trace equivalence $\left(\simeq_{T}\right)$, which also provides an inductive principle to use in the proof. The replacement is possible because the two equivalences have been proved to coincide in $\mathcal{A}+1$ [Patrignani and Clarke 2014]. This direction is thus restated as $C_{1} \simeq C_{2} \Rightarrow C_{1}^{\downarrow} \simeq_{\mathrm{T}} C_{2}^{\downarrow}$.

Trace equivalence is based on trace semantics, which describes the behaviour of a program in terms of a set of traces: sequences of actions the program can undertake [Jeffrey and Rathke 2005b]. Two programs are thus trace equivalent if their trace semantics coincide, i.e. if their sets of traces are the same. This notion (or rather, its negation) is used to prove the contrapositive of this direction: $C_{1}^{\downarrow} \neq_{\mathrm{T}} C_{2}^{\downarrow} \Rightarrow C_{1} \neq C_{2}$.

In order to prove that $C_{1}$ and $C_{2}$ are not contextually equivalent, it suffices to show that there exists a source-level context that behaves differently depending on whether its hole is filled with $C_{1}$ or $C_{2}$. Such a context is said to differentiate $C_{1}$ from $C_{2}$. This proof relies on an algorithm that creates a source-level context, a witness that differentiates $C_{1}$ from $C_{2}$, given the different traces of their compiled counterparts $C_{1}^{\downarrow}$ and $C_{2}^{\downarrow}$.

This proof strategy is known in the field [Agten et al. 2012; de Boer et al. 2005; Jeffrey and Rathke 2005a; Patrignani and Clarke 2014]. However, the complexity of the proof resides in handling features of the high-level language such as strong typing, dynamic memory allocation and exceptions.

\subsection{Contextual Equivalence: a Security Perspective}

Contextual equivalence plays a key rôle in the definition of a fully abstract compiler, yet what ensures that a fully abstract compiler is a secure one? This section briefly explains how contextual equivalence can be used for security policies enforcement [Abadi 1999; Abadi and Plotkin 2012; Agten et al. 2012; Ahmed and Blume 2011; Fournet et al. 2013; Jagadeesan et al. 2011; Kennedy 2006; Patrignani et al. 2013].

In $\mathcal{J}+E$, fields are private, so every allocated object defines a secret state: the contents of its fields. Some objects can thus be indistinguishable from an external point of view even though their states differ: they are contextually equivalent.

Contextual equivalence can be adopted to state security properties such as confidentiality, integrity and invariant definition, as the code examples of Fig. 3 show [Abadi and Plotkin 2012; Agten et al. 2012]. In the examples, classes are annotated with 
Objects $o_{L}$ and $o_{R}$ are equivalent at the source level, but their compiled counterparts are not. Since local variables are placed on the call stack (in unprotected memory) and a low-level attacker can read unprotected memory, she can read the value of $x$ during the callback cb.callback(). Variable $x$ contains the value of secret, which is a private field and which is different for both objects.

A naïve compilation scheme does not entail the confidentiality or integrity of the call stack, which allows attackers to read and write local variables. An attacker can use this vulnerability to read secrets from the stack, similarly to a buffer-overread attack [Strackx et al. 2009]. Alternatively she can even tamper with the control flow by overwriting a return address on the stack, similarly to a return address clobbering attack [Erlingsson et al. 2010].

The second point ensures that the only way to divert the flow of execution is through exceptions. Since exception throwing must be specified in method interfaces, critical functionalities can be implemented so that they are carried out in their entirety: if the right exceptions are caught, no other way of diverting the execution flow exists.

The third point is similar to the second one: there is no way of disrupting some functionalities by supplying arbitrary parameters to method calls.

The presence of a protected module provides a basic protection of some of the aforementioned properties, but not all of them are covered. The adoption of a secure compiler for the software to be placed in the protected memory should ensure that all aforementioned security properties are enforced in that software. By defining such a secure compiler as a fully abstract compiler, we capture exactly the preservation of those properties in the generated target code: a fully abstract compiler makes the software in the protected memory secure.

For a more precise treatment, the threat model consists of the following definitions: the system under attack (Definition 2.4), the security property of the system (Definition 2.5), and the attacker to the system (Definition 2.6).

Definition 2.4 (System under attack). The system is a Von Neumann machine with a flat address space and one PMA instance that provides a single protected partition in memory (a protected module). The protected module contains $\mathcal{A}+1$ code, called the protected code, that complies to $\mathcal{J}+\mathrm{E}$ specifications, so the protected code behaves like $\mathcal{J}+$ E code. The unprotected memory contains arbitrary $\mathcal{A}+$ l code.

In the system under consideration, for the sake of simplicity, only compiled $\mathcal{J}+\mathrm{E}$ software is considered to be present. Moreover, only one protected module is assumed to be present. A single module suffices to protect the concerns of a single user or of multiple users that trust each other. Addressing the challenges of adopting multiple protected modules in the system, each belonging to mutually-distrusting stakeholders, is left for future work.

Definition 2.5 (Security property). The protected code behaves as its $\mathcal{J}+$ E specifications and in no other way.

This property has the security implications described above since it is applied to the $\mathcal{J}+\mathrm{E}$ language. In different languages, this property may not have the same security relevance. For example, this property in the context of the $\mathrm{C}$ language would not entail confidentiality and integrity, as any structure can be inspected by virtue of simple pointer arithmetic. ${ }^{4}$

\footnotetext{
${ }^{4}$ The $\mathrm{C}$ standard states that the behaviour of these scenarios is "undefined", but most $\mathrm{C}$ compilers allow
} arbitrary pointer arithmetic. 
Definition 2.6 (Attacker). The attacker can arbitrarily change the state of the unprotected partition of the same memory of the protected module. The attacker also knows how to interact with the secure module.

The attacker is assumed to know the interfaces implemented by the protected code: the location of each entry point, the types each function expects and the addresses of eventual static objects. Knowledge of the functionalities of existing software in the system is given to the attacker so the injected code can interact (possibly safely) with existing software.

Limitations. This threat model does not cover all possible security threats that the system is subject to, as exemplified below. Source-level security violations, e.g. a method returning a field-stored private key that was supposed to be secret, are not considered in this paper. These violations should not be countered at the compiler level, but with source-level artefacts such as type systems. Availability attacks, e.g. unprotected code that never calls protected code, are also not considered, since the attacker is assumed to interact with the software to be protected in order to violate its security properties. Finally, side channel attacks are also not considered. The definition of the attacker's power, in fact, limits the kind of attacks she can mount. The attacker cannot exploit covert channels to mount side-channels attacks such as timing attacks, since these attacks cannot be expressed with $\mathcal{A}+$ l code.

This paper now describes how to develop a secure compiler that counters the threat imposed by the aforementioned attacker.

\section{SECURE COMPILATION OF $\mathcal{J}+\mathrm{E}$}

This section firstly describes the general structure of the secure compilation scheme for $\mathcal{J}+E$. Then it presents details of the secure compiler in three parts, all of which introduce a series of naïve mistakes to secure compilation of a certain feature and then correct them. The first feature to be presented is callbacks (Section 3.1), the second one is dynamic memory allocation (Section 3.2) and the final one is exceptions (Section 3.3).

For the sake of simplicity, we start by developing a secure compiler for a core of $\mathcal{J}+\mathrm{E}$. In the following, assume no dynamic memory allocation (i.e. no new expressions) and no presence of exceptions (i.e. no try/catch blocks and no throw $e$ expressions). Since no new objects are created at runtime, components use static objects and externs for now.

We follow some standard conventions about how objects are compiled [Ducournau 2011]. The compilation of a $\mathcal{J}+E$ component $C$ outputs a protected module $C^{\downarrow}$, written in $\mathcal{A}+\mathrm{l}$, consisting of a partial memory space and a memory descriptor. The program $C^{\downarrow}$ interacts with should not be able to distinguish modules just by their size, so, a constant amount of memory is reserved for each protected module, independent of the actual memory space required. The protected module is placed in protected memory and the memory descriptor divides the reserved space over the code and the data section. The stack pointer register is set up by the context and is pointing to free space in unprotected memory.

The compilation process consists of translating each package, class, object, interface and method of the input component. To prevent a low-level module from being distinguished by the order of its methods in memory, packages, interfaces, methods in interfaces, classes, objects and externs are sorted alphabetically. Methods that do not appear in interfaces are compiled based on the order of occurrence in the class.

When an object is compiled, a word is reserved to indicate its class, which is used to dynamically dispatch methods. Methods are dispatched based on offsets through the v-tables, which associate class and method offsets with the corresponding method 
body. For each object, fields are then given a unique index number starting at 1 , based on their order of occurrence. For a field $f_{i}$, one word of memory is reserved at the $i$ th memory address of the memory section of a given object. Integer-typed constants are translated to their corresponding numeric value, unit is translated to 0 , true and false are translated to 1 and 0 respectively.

To translate a method body, the compiler processes each expression in turn, translating it into a list of behaviourally-equivalent instructions. Registers $r_{0}$ to $r_{3}$ are used as general working registers, return values are passed through $r_{0}$. In a method call at the target level, register $r_{4}$ identifies the current object (this). Method calls are limited to seven parameters, which are passed through registers $r_{5}$ to $r_{11}$. These choices are not critical, the compiler may use registers in a different way and still be fully abstract. A calling convention is set so that register $r_{0}$ contains the address where to return after a call at the target level (i.e. any jump instruction between the protected and unprotected sections, located at address $x$ is preceded by an instruction movi $r_{0} x+1$ ). This restriction simplifies the proof of full abstraction of the compilation scheme, we envisage it can be lifted at the price of complicating the proof, without making it unprovable.

Parameters and local variables are given a method-local index number. For each translated method body, a prologue and an epilogue are prepended and appended to it. The prologue allocates and initialises a new activation record on the call stack, the record contains local variables and parameters for the method body. The epilogue deallocates the activation record when the method is done. The code of prologues, epilogues and method bodies is placed in free space in the code section.

To support programming to an interface, and since protected memory can be entered only through entry points, a method entry point is generated for each interface-defined method. The entry point for the $i$-th method is placed at address $i * 128$ of the code section. The offset of 128 memory words is chosen arbitrarily, with the only condition that there is enough space between entry points to perform a number of simple operations, as will be described in Section 3.1. Each entry point forwards the call to the actual method body, so code at entry points consists of two parts: (1) a call to the method's body and (2) a return instruction. When the call to the body returns, the return instruction returns control to the location from which the entry point was called.

In order to specify how the component interacts with external code, assume the component being compiled provides one import package without a corresponding export one. Refer to this package as the distinguished import package (DIP). The DIP contains interface and extern definitions, thus callbacks are calls to methods defined in the DIP, on externs defined in the DIP. Component code is not supposed to implement interfaces defined in the DIP, as those are functionalities it requires of external programs. External code which provides an implementation for the DIP can implement interfaces defined in the component. This can lead to the dynamic dispatch procedure being called on objects that are not in the protected memory partition. When this case is detected, the compiled component must behave as in the case of a callback. The address where protected code must jump when performing a callback is assumed to be known based on a calling convention set up between protected and unprotected code.

So, a return entry point is generated to support returning from a callback. To perform a callback, the actual return address is first pushed on the call stack, then the address of the return entry point is pushed on the call stack. Control is then transferred to unprotected memory. When the code in unprotected memory returns from the callback, control will first be transferred to the return entry point, which will then subsequently return back to the actual return address. 
The compilation scheme as described above ensures that a module is exited either through a callback, or through the return statement at the end of an entry point. Therefore, the second part of each method entry point is named exit point.

To provide a better understanding of the compilation scheme thus far, Fig. 4 presents the memory layout of the compiled counterpart of the code of Listing 1.

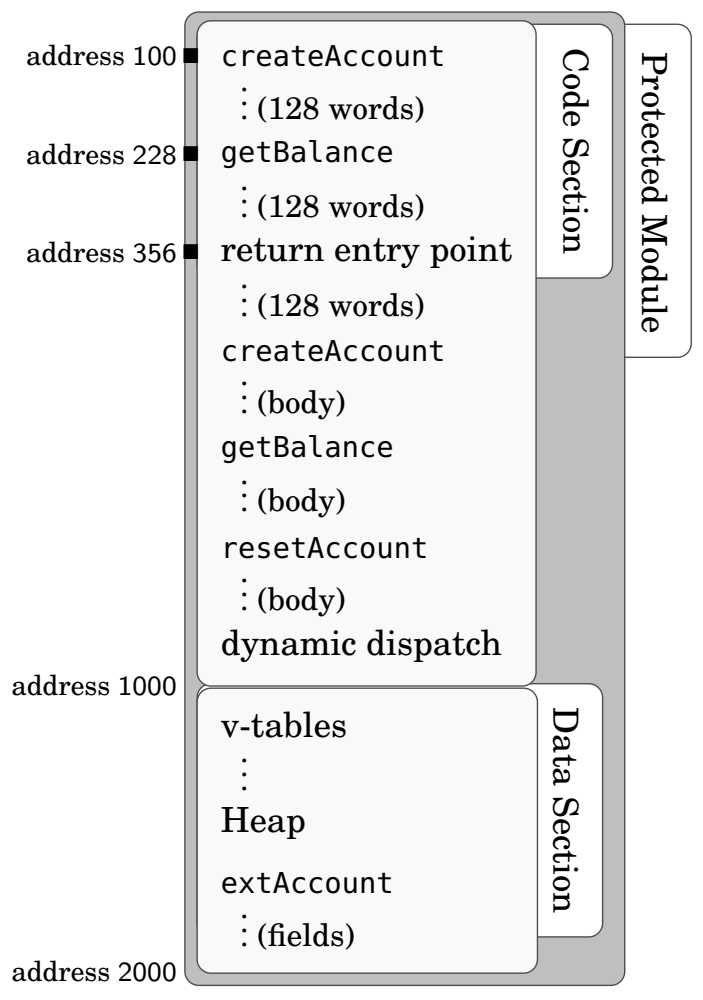

Fig. 4: Memory layout of the compilation of code from Listing 1 . $\square$ indicate entry points. The protected memory partition spans from address 100 to address 2000 , the code section spans for 900 addresses.

\subsection{Secure Compilation of Callbacks}

The compilation scheme places code and data of the compiled object in the protected memory partition and configures the entry points of the protected memory partition to forward calls to method implementations. This scheme is sound, in the sense that two nonequivalent $\mathcal{J}+\mathrm{E}$ components will be compiled into two nonequivalent $\mathcal{A}+\mathrm{I}$ programs. It also provides some basic protection; for instance, a target-level attacker cannot just scan memory to find the values of object fields as this is prevented by the protected module architecture. However this scheme fails to be secure, this is shown by means of counterexamples.

3.1.1. Limitations of the Compilation Scheme. The compilation scheme defined so far is not fully abstract, as illustrated by the series of examples below; Problem 1 also arises in this context. In these examples, $\mathcal{A}+\mathrm{I}$ modules can be differentiated, while their $\mathcal{J}+\mathrm{E}$ 
The secure stack is given a large enough upper bound so that most programs can run without overflowing it. If the stack is overflowed, all registers and flags are cleared and the execution halts.

At the start of each entry point, the stack is switched to the secure stack. When leaving the protected module, the stack is restored to its previous address, which is checked to be in unprotected memory. When returning from protected to unprotected code, the return address is also checked to be in unprotected memory. A callback is performed by first pushing the actual return address onto the secure stack. Then, $r_{4}$ is stored in the secure stack so as to be able to restore this to the right value once the callback returns. Next, the stack is switched to the unprotected stack and the address of the return entry point is pushed onto it. Control is then transferred to the context. When the callback returns, control will first be transferred to the return entry point, which switches back to the secure stack. Then it restores this to the expected value and it transfers control back to the actual return address. Because data is written to the unprotected stack during this process, the compiler must ensure that the location of the unprotected stack is valid before it writes to it. That is, the address of the unprotected stack must lie outside of protected memory, for otherwise parts of protected memory might get overwritten.

The context could tamper with the control flow by jumping to the return entry point when there is no callback to return from. To prevent this, the compiler initialises the first location of the secure stack to the address of a procedure clears all registers and flags and then halts. The return entry point will jump to this address if it is called when there is no callback to return from.

Information leakage. In $\mathcal{J}+\mathrm{E}$, the only way for two objects to communicate, is through well-typed method calls and returns. The compiler must ensure that a low-level attacker cannot use any other communication channels, as this might leak information that should be kept private to the protected module.

The model of $\mathcal{A}+1$ inherently provides three ways to exchange information: (1) through unprotected memory, (2) through the flags register and (3) through the general purpose registers $r_{0}$ to $r_{11}$ and SP. Method (1) is already precluded, as compiled programs never write in unprotected memory. The SP register does not convey private information, because it is restored to the location of the unprotected stack whenever control leaves the protected module. The compiler constrains methods (2) and (3) as follows:

— Flags are cleared at each callback and exit point.

- Every general purpose register except $r_{0}$ is cleared at each exit point.

- Every general purpose register is cleared at each callback, except if it is used for passing a parameter.

The compiler generates code at each entry point to enforce these constraints.

Value of booleans. The compiler must ensure that all memory locations corresponding to $\mathcal{J}+\mathrm{E}$ fields and variables contain only values for which there is a corresponding $\mathcal{J}+\mathrm{E}$ value. The only values of type Bool are true and false and the corresponding $\mathcal{A}+1$ values are 1 and 0 . The compiler enforces this constraint by adding a run-time check at each entry point, to verify that the value of any Bool-typed parameter is either 0 or 1. An analogous check is added at each callback to a method with return type Bool. The same checks are introduced for type Unit, inhabited by unit in $\mathcal{J}+\mathrm{E}$ which is compiled to 0 in $\mathcal{A}+$ l. If any check fails, all registers and flags are cleared and the execution halts. 
deterministic order, based on the interaction between external and internal code. Code at entry points is responsible for retrieving object identities from $\mathcal{O}$ before the actual method call. Access and retrieval of entries in $\mathcal{O}$ is very fast and it can be implemented in $O(1)$. As the only objects in the data structures are the ones the attacker knows, she cannot guess object identities. This does not hamper the functionality of external code as it can only call methods on objects.

Consider for example the right code snippet in Problem 6. There, $o_{R} 1$ and $o_{R} 2$ are given index 0 and 1 and they are added in $\mathcal{O}$ at compile time (since they are static objects). Once method createSecret is called, two objects are created. However, the object saved in variable $\mathrm{x}$ is not returned, so it is not added to $\mathcal{O}$. The other object is, so it is added to $\mathcal{O}$. Thus, at the $\mathcal{A}+\mathrm{l}$ level, the compiled counterpart of createSecret will return 2 the first time it is called in both $\mathrm{p}_{L}$ and $\mathrm{p}_{R}$.

Entry points. Table II describes the code executed at entry points. Both method entry

\begin{tabular}{|c|c|c|c|}
\hline \multicolumn{2}{|r|}{ Method $p$ entry point } & \multicolumn{2}{|r|}{ Preamble to returnback entry point } \\
\hline 1 & Load receiver $v=\mathcal{O}\left(\mathrm{r}_{4}\right)$ & $\mathrm{a}$ & Push current object $v=\mathrm{r}_{4}$, return \\
\hline 2 & Check that v's class defines & $\mathrm{b}$ & Reset flags and unused registers \\
\hline 3 & Load parameters $\bar{v}$ from $\mathcal{O}$ & c & $\begin{array}{l}\text { Replace object identities with in- } \\
\text { dexes in } \mathcal{O}\end{array}$ \\
\hline 4 & $\begin{array}{l}\text { Dynamic typecheck on } \bar{v} \\
\text { Perform dynamic dispatch } \\
\text { (run method p code) }\end{array}$ & d & $\begin{array}{l}\text { Jump to callback address } \\
\text { (run external code) }\end{array}$ \\
\hline \multicolumn{2}{|r|}{ Exit point } & \multicolumn{2}{|r|}{ Returnback entry point } \\
\hline 6 & Reset flags and unused registers & & and check it \\
\hline \multirow[t]{2}{*}{7} & Replace object identities with in- & $\mathrm{f}$ & \\
\hline & & $\mathrm{g}$ & $\begin{array}{l}\text { Pop return address } a \text {, current ob- } \\
\text { ject } v \text { and resume execution }\end{array}$ \\
\hline
\end{tabular}

Table II: Extension to the pseudocode executed at entry points presented in Table I. Loading means that a value is retrieved from the memory, push and pop are operations on the secure stack.

points and the return entry point are logically divided in two parts; they maintain the functionalities introduced in Section 3.1 and expand them as follows. The first part performs the checks described below and then jumps either to the code that performs the dynamic dispatch or to the callback. The second part returns control to the location from which the entry point was called.

For method calls to be well-typed, the code at entry points performs dynamic typechecks. This checks that a method is invoked on objects of the right type (line 2), with parameters of the right type (line 4), addressing Problem 4 and 5. Similar checks are executed when returning from a callback, in the returnback entry point (line f). These checks are performed only on objects whose class is defined in the compiled component, as they are allocated in protected memory; no control over externally allocated objects can be assumed. Dynamic typechecks are performed based on the word that indicates the class of a compiled object, that value is checked to comply with the value the method expects.

Resetting flags and registers are as in Section 3.1. If any check fails, all registers and flags are cleared and the execution halts. 
A convention between protected and unprotected code is needed in order to identify indexes in $\mathcal{O}$ from unprotected addresses. For this, assume that the leftmost bit of a word is 1 if it denotes an index in $\mathcal{O}$.

\subsection{Secure Compilation of Exceptions}

To present secure compilation of exceptions, from this section onward, source-level components can contain try/catch blocks and throw $e$ expressions. Method signatures can specify if the method throws a particular exception.

Secure compilation of languages supporting exceptions must handle the difficulties that result from the modification of the flow of execution of a program. This can be modified when a part of a program throws an exception and another part catches it. Exception handling can be implemented by modifying the runtime of the language so that it knows where to dispatch a thrown exception. Activation records are responsible for pointing to the exception handlers in order to propagate a thrown exception to the right handler.

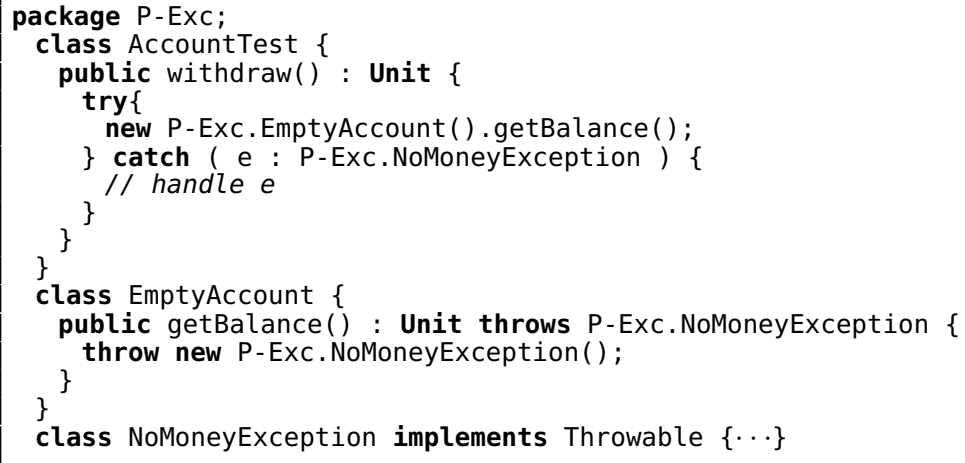

Listing 2: Example of exceptions usage.

In Listing 2, the catch block of method withdraw ( ) in class AccountTest defines a handler for exceptions of type NoMoneyException. When the activation record for withdraw( ) is allocated, the handler is registered in the related allocation record. When an exception of type NoMoneyException is thrown, the stack is traversed to find the closest handler for exceptions of type NoMoneyException. As activation records are traversed and a handler is not found, those records are popped from the stack.

In order to implement throwing an exception in secure code that is caught in insecure code (or vice versa), throwing is securely compiled as callbacks (or calls). Thus two additional entry points are created: the throw entry point and the throwback entry point. These entry points forward calls to the secure and insecure exception dispatchers, respectively. The secure exception dispatcher traverses the secure stack looking for handlers for the thrown exception. After an activation record has been inspected and deallocated, if the 'next' allocation record to be inspected is in unprotected memory, the exception is forwarded to the external code through the throwback entry point.

Since exceptions are objects, in order to prevent exploits as in Problem 4, the throwback entry point must remember internally allocated exceptions that are thrown to external code. So, data structure $\mathcal{O}$ is used to register leaked exceptions as well as leaked object identities. This prevents external code from passing a non-existing object identity to the secure exception handler in place of the object identity of an exception, effectively throwing a non existent exception. 
For protected code to catch unprotected exceptions (and vice-versa) based on the (unprotected) interface type declared by the thrown object, some modifications are needed. A table is added in the protected code section, where unprotected object ids are associated to their known interface types. When an unprotected object is made known to the protected code (as a parameter in a call or by being returned), the object id is added to the table, alongside the interface types it is known to implement. So, when a catch block is defined for an unprotected interface type, the type information of unprotected objects is known and, in case an unprotected object is thrown, it can be caught.

Fig. 5 presents a graphical overview of how exceptions are handled normally (on the left) and in the presented compilation scheme (on the right). Lower case letters
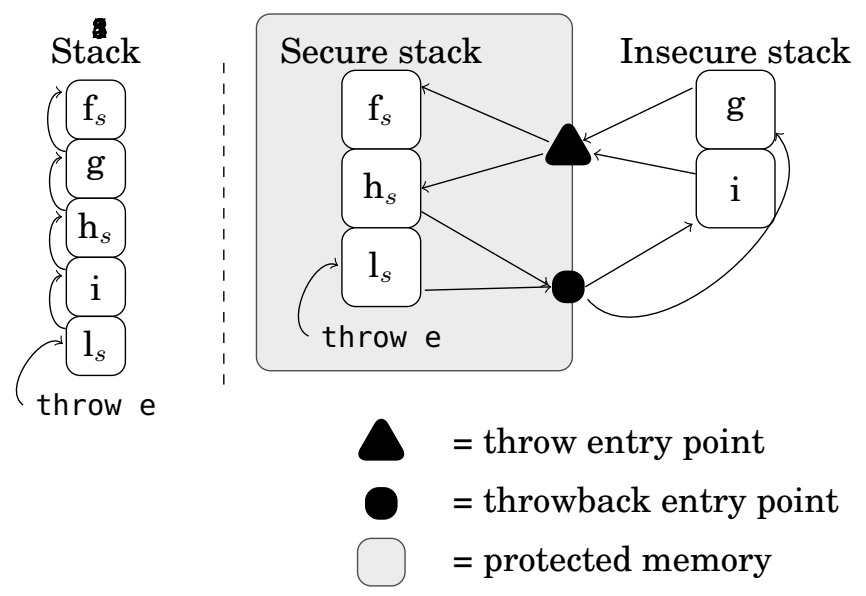

Fig. 5: Comparison of ways to handle exceptions.

indicate the allocation record for the corresponding function. A subscript $s$ indicates a secure function; the stack grows downward. The order in which exception handlers are searched is indicated on arrows. The throw and throwback entry point split the same arrow in two parts.

The introduction of two additional entry points may seem to introduce functionality at the target level that the source-level lacks; however this is not the case. Only exceptions of existing types can be thrown and handling exceptions follows the normal course of the stack. The external code could replace an exception, but this is equivalent to the high-level language functionality to catching an exception and throwing another one. Thus the target level is not granted additional functionality.

3.3.1. Limitations of the Compilation Scheme. In the context of secure compilation, if exception handlers are compiled as above, one problem arises.

Problem 7 (EXCESSIVE EXCEPTION CATCHING). Consider two methods safeCallback that invoke method callback on an input parameter. Even though callback does not specify that it will throw exceptions, one implementation of safeCallback wraps it in a try/catch block. 


$\begin{array}{lrlrl}\text { Words } & w & :=[0 \text { or } 1]^{\ell} & \text { Memories } & m::=\emptyset \\ \text { Instructions } & i & \in \mathcal{I} \subset \text { Words } & & \mid m ; a \mapsto w \\ \text { Empty word } & \mathbf{0}::=0^{\ell} & \text { Numbers } & n::=n \in \mathbb{N} \\ \text { Addresses } & a & \in 0 . .2^{\ell}-1 & \text { Programs } & P::=(m, s) \\ \text { Memory descriptors } & s::=\left(a_{b}, n_{c}, n_{d}, n\right) & & \end{array}$

Fig. 6: Elements of the formalisation of $\mathcal{A}+$.

empty word 0 is a sequence of 0's whose length is based on the architecture being considered. Addresses $a$ are natural numbers, ranging from 0 to $2^{\ell}-1$. Memories $m$ are maps from addresses to words. Memory access, denoted as $m(a)$, is defined as follows: $m(a)=w$ if $a \mapsto w \in m$; it is undefined otherwise. Define the domain of a memory as $\operatorname{dom}(m)=\{a \mid a \mapsto w \in m\}$. If two memories $m$ and $m^{\prime}$ have disjoint domains, they can be merged to yield another memory. Formally, if $\operatorname{dom}(m) \cap \operatorname{dom}\left(m^{\prime}\right)=\emptyset$, then $m+m^{\prime}=\left\{a \mapsto w \mid a \mapsto w \in m\right.$ or $\left.a \mapsto w \in m^{\prime}\right\}$. Memory descriptors $s$ are quadruples: $\left(a_{b}, n_{c}, n_{d}, n\right): a_{b}$ is the address where the protected memory partition starts, $n_{c}$ and $n_{d}$ are the sizes (in number of addresses) of the code and data section respectively and $n$ is the number of entry points. Entry points are allocated starting from the base address $a_{b}$. Each entry point is $\mathcal{N}_{e}$ words long (in the secure compilation scheme, $\mathcal{N}_{e}$ is 128). Assume the entry points do not overflow the protected code section, thus the constraint $n \cdot \mathcal{N}_{e}<n_{c}$ holds for the all memory descriptors. Programs $P$ are pairs of a memory $m$ and a memory descriptor $s$.

4.1.2. Semantics. Before introducing the semantics, Fig. 7 defines the memory access control enforcement rules. Read judgments $s \vdash$ predicate $(a, b, \cdots)$ as: "according to $s$, predicate holds for addresses $a, b, \ldots$ ”. Whenever an access control rule is violated by

$$
\begin{aligned}
& \begin{array}{ll}
\text { (Aux-protected) } & \text { (Aux-unprotected1) }
\end{array} \\
& \frac{a_{b} \leq p<\left(a_{b}+n_{c}+n_{d}\right)}{s \vdash \operatorname{protected}(p)} \quad \frac{p<a_{b}}{s \vdash \operatorname{unprotected}(p)} \quad \frac{\left(a_{b}+n_{c}+n_{d}\right) \leq p}{s \vdash \operatorname{unprotected}(p)} \\
& \text { (Aux-returnEntry) (Aux-entryPoint) (Aux-data) } \\
& \begin{array}{cccc}
p=a_{b}+(n-1) \cdot \mathcal{N}_{e} & & p=a_{b}+m \cdot \mathcal{N}_{e} & \left(a_{b}+n_{c}\right) \leq p \\
s \vdash \operatorname{returnEntryPoint}(p) & \frac{m \in \mathbb{N} m<n}{s \vdash \operatorname{entryPoint}(p)} & \frac{p<\left(a_{b}+n_{c}+n_{d}\right)}{s \vdash \operatorname{data}(p)}
\end{array}
\end{aligned}
$$

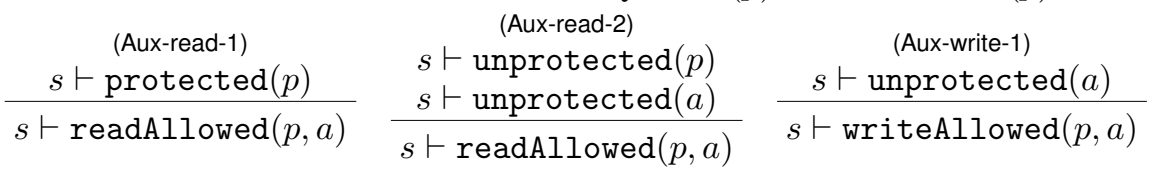

$$
\begin{aligned}
& \text { (Aux-write-2) (Aux-entry) (Aux-return) } \\
& s \vdash \operatorname{protected}(p) \quad s \vdash \operatorname{unprotected}(p) \quad s \vdash \operatorname{protected}(p) \\
& \frac{s \vdash \operatorname{data}(a)}{s \vdash \operatorname{writeAllowed}(p, a)} \frac{s \vdash \operatorname{entryPoint}\left(p^{\prime}\right)}{s \vdash \operatorname{entry} \operatorname{Jump}\left(p, p^{\prime}\right)} \frac{s \vdash \operatorname{unprotected}\left(p^{\prime}\right)}{s \vdash \operatorname{exitJump}\left(p, p^{\prime}\right)} \\
& \text { (Aux-internal) } \\
& s \vdash \operatorname{protected}(p) \quad s \vdash \operatorname{unprotected}(p) \\
& \frac{s \vdash \operatorname{protected}\left(p^{\prime}\right) \quad s \nvdash \operatorname{data}\left(p^{\prime}\right)}{s \vdash \operatorname{int} \operatorname{Jump}\left(p, p^{\prime}\right)} \quad \frac{s \vdash \operatorname{unprotected}\left(p^{\prime}\right)}{s \vdash \operatorname{ext} \operatorname{Jump}\left(p, p^{\prime}\right)}
\end{aligned}
$$

Fig. 7: Access control enforcement rules. Assume $s \equiv\left(a_{b}, n_{c}, n_{d}, n\right)$ 
a program, the execution is halted and the program counter is set to -1 .

Define functions $\mathrm{m}_{\mathrm{sec}}(m, s)$ and $\mathrm{m}_{\mathrm{ext}}(m, s)$, which return the protected and unprotected parts of a memory $m$ according to the descriptor $s$, respectively as: $\mathrm{m}_{\mathrm{sec}}(m, s)=$ $\{a \mapsto w \mid a \mapsto w \in m, s \vdash \operatorname{protected}(a)\}$ and $\mathrm{m}_{\text {ext }}(m, s)=\{a \mapsto w \mid a \mapsto w \in m, s \vdash$ unprotected $(a)\}$.

The operational semantics is a small step semantics that describes how each instruction of the language transforms an execution state into a new one. Thus, the operational semantics handles programs in the whole memory: both the protected and unprotected partitions.

Definition 4.1 (Execution state). An execution state, denoted as $\Omega$, is a quintuple $\Omega=(p, r, f, m, s)$, where $p$ is a program counter, $r$ is a register file, $f$ is a flags register, $m$ is a memory and $s$ is a memory descriptor.

Given $\Omega=(p, r, f, m, s)$, let $\lfloor\Omega\rfloor$ be the state which encompasses only the protected memory: $\left(p, r, f, \mathrm{~m}_{\mathrm{sec}}(m, s), s\right)$. Analogously, let $\lceil\Omega\rceil$ be the state that encompasses only the unprotected memory: $\left(p, r, f, \mathrm{~m}_{\text {ext }}(m, s), s\right)$. Relations $\stackrel{i}{\rightarrow} \subseteq\lfloor\Omega\rfloor \times\lfloor\Omega\rfloor$ and $e_{\subseteq} \subseteq\lceil\Omega\rceil \times\lceil\Omega\rceil$ describe the evaluation of instructions that only affect the protected and unprotected parts of memory respectively. Fig. 8 presents the rules for $\stackrel{i}{\rightarrow}$, rules for $e_{\rightarrow}$ are obtained by replacing an int Jump assumption with an ext Jump one. In the rules, notation $m[a \mapsto w]$ indicates that memory $m$ is updated to a new one that is equal to $m$ except that the value stored at address $a$ is $w$. Notation $r[\mathrm{R} \mapsto w]$ indicates that the register file $r$ is updated to a new one that is equal to $r$ except that the value stored in register $\mathrm{R}$ is $w$. Notation $r(\mathrm{R})$ indicates the value contained in register $\mathrm{R}$ in register file $r$. Let $m(p)=$ inst denote that inst is the word allocated in $m(p)$, where inst $\in \mathcal{I}$. Note that the program counter is set to -1 whenever the halt instruction is encountered, in order to capture termination. This way, no progress can be made, as $m(-1)$ does not return a valid instruction: the program is in a stuck state.

Definition 4.2 (Stuck state). A state $\Omega=(p, r, f, m, s)$ is stuck, denoted as $\Omega^{\perp}$, when the program counter does not point to a valid instruction: $m(p) \notin \mathcal{I}$.

The operational semantics of $\mathcal{A}+\mathrm{l}$ is a binary relation over states $\rightarrow \subseteq \Omega \times \Omega$ defined by the rules of Fig. 9. It relies on relations $\stackrel{i}{\rightarrow}$ and $e_{\rightarrow}$, for transitions that affect only a section of memory, as captured by rules Eval-protected and Eval-unprotected. The compiler ensures that rules Eval-movl-out and Eval-movs-out will never be executed and that unused registers and flags are always reset to 0 when jumping between protected and unprotected memory sections. Rule Eval-callback (and return) is performed when executing a callback (and a return). The only difference between the callback and the return cases is that in the latter one, all registers besides $r_{0}$ contain 0 .

The transitive closure of relation $\rightarrow$ is indicated with $\rightarrow$. A state $\Omega$ performing $n$ reduction steps is indicated as $\Omega \rightarrow^{n} \Omega^{\prime}$. The evaluation of program $P$ is a sequence of steps that takes the initial state of $P$ to another state.

Definition 4.3 (Initial state). The initial state of a program $(m, s)$, denoted as $\Omega_{0}(m, s)$, is the state $\left(p_{0}, r_{0}, f_{0}, m, s\right)$, where $s=\left(a_{b}, n_{c}, n_{d}, n\right), p_{0}=\left(a_{b}+n_{c}+n_{d}+2\right)$, $r_{0}=\left[\mathrm{SP} \mapsto 0 ; r_{\mathrm{i}} \mapsto 0_{\mathrm{i}=0 . .11}\right]$, and $f_{0}=[\mathrm{ZF} \mapsto 0 ; \mathrm{SF} \mapsto 0]$.

The evaluation of $P$ terminates if $\Omega_{0}(P) \rightarrow^{*} \Omega^{\perp}$; the result of the computation is stored in $r_{0}$. If the evaluation of program $P$ does not terminate, $P$ diverges. A program $P$ diverges, denoted as $P \Uparrow$, if it executes an unbounded number of reduction steps. Formally: $P \Uparrow$ if $\forall n \in \mathbb{N}, \exists \Omega^{\prime} . \Omega_{0}(P) \rightarrow^{n} \Omega^{\prime}$.

Fully abstract compilation relies on the notion of contextual equivalence, which is now defined. Contextual equivalence relates two programs that cannot be distin- 


$$
\begin{aligned}
& \text { (Eval-movl) } \\
& m(p)=\left(\operatorname{mov} l r_{\mathrm{d}} \mathrm{r}_{\mathrm{s}}\right) \\
& s \vdash \operatorname{int} \operatorname{Jump}(p, p+1) \\
& s \vdash \operatorname{readAllowed}\left(p, r\left(\mathrm{r}_{\mathrm{s}}\right)\right) \\
& r^{\prime}=r\left[r_{\mathrm{d}} \mapsto m\left(r\left(\mathrm{r}_{\mathrm{s}}\right)\right)\right] \\
& (p, r, f, m, s) \stackrel{i}{\rightarrow}\left(p+1, r^{\prime}, f, m, s\right) \\
& \text { (Eval-movi) } \\
& m(p)=\left(\text { movi } r_{\mathrm{d}} \mathrm{i}\right) \\
& s \vdash \operatorname{int} \operatorname{Jump}(p, p+1) \\
& r^{\prime}=r\left[\mathrm{r}_{\mathrm{d}} \mapsto i\right] \\
& (p, r, f, m, s) \stackrel{i}{\rightarrow}\left(p+1, r^{\prime}, f, m, s\right) \\
& \text { (Eval-add) } \\
& m(p)=\left(\operatorname{add} r_{\mathrm{d}} \mathrm{r}_{\mathrm{s}}\right) \\
& s \vdash \operatorname{int} \operatorname{Jump}(p, p+1) \\
& v=\left(r\left(\mathrm{r}_{\mathrm{d}}\right)+r\left(\mathrm{r}_{\mathrm{s}}\right)\right) \% 2^{\ell} \quad r^{\prime}=r\left[\mathrm{r}_{\mathrm{d}} \mapsto v\right] \\
& f^{\prime}=f[\mathrm{ZF} \mapsto(v==0)] \\
& (p, r, f, m, s) \stackrel{i}{\rightarrow}\left(p+1, r^{\prime}, f^{\prime}, m, s\right) \\
& \text { (Eval-function-call) }
\end{aligned}
$$

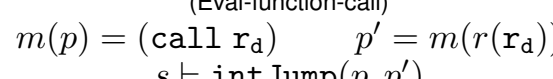

$$
\begin{aligned}
& s \vdash \operatorname{int} \operatorname{Jump}\left(p, p^{\prime}\right) \\
& r^{\prime}=r[\mathrm{SP} \mapsto r(\mathrm{SP})+1] \\
& m^{\prime}=m\left[r^{\prime \prime}(\mathrm{SP}) \mapsto p+1\right] \\
& (p, r, f, m, s) \stackrel{i}{\rightarrow}\left(p^{\prime}, r^{\prime}, f, m^{\prime}, s\right) \\
& \text { (Eval-je-true) } \\
& m(p)=\left(\begin{array}{ll}
\text { je } r_{\mathrm{i}}
\end{array}\right) \quad f(\mathrm{ZF})==1 \quad m(p)=\left(\mathrm{jl} \mathrm{r}_{\mathrm{i}}\right) \quad f(\mathrm{SF})==1 \\
& \frac{p^{\prime}=r\left(r_{\mathrm{i}}\right) \quad s \vdash \operatorname{int} \operatorname{Jump}\left(p, p^{\prime}\right)}{(p, r, f, m, s) \stackrel{i}{\rightarrow}\left(p^{\prime}, r, f, m, s\right)} \quad \frac{p^{\prime}=r\left(r_{\mathrm{i}}\right) \quad s \vdash \operatorname{int} \operatorname{Jump}\left(p, p^{\prime}\right)}{(p, r, f, m, s) \stackrel{i}{\rightarrow}\left(p^{\prime}, r, f, m, s\right)} \\
& \text { (Eval-je-false) } \\
& m(p)=\left(\text { je } r_{\mathrm{i}}\right) \quad f(\mathrm{ZF})==0 \\
& s \vdash \operatorname{int} \operatorname{Jump}(p, p+1) \\
& \overline{(p, r, f, m, s) \stackrel{i}{\rightarrow}(p+1, r, f, m, s)} \quad \overline{(p, r, f, m, s) \stackrel{i}{\rightarrow}(p+1, r, f, m, s)} \\
& \text { (Eval-jump) } \\
& \frac{m(p)=\left(j m p r_{\mathrm{d}}\right) \quad p^{\prime}=r\left(r_{\mathrm{d}}\right) \quad s \vdash \operatorname{int} \operatorname{Jump}\left(p, p^{\prime}\right)}{(p, r, f, m, s) \stackrel{i}{\rightarrow}\left(p^{\prime}, r, f, m, s\right)} \\
& \text { (Eval-jl-false) } \\
& s \vdash \operatorname{int} \operatorname{Jump}\left(p, p^{\prime}\right) \\
& \frac{r^{\prime}=r[\mathrm{SP} \mapsto r(\mathrm{SP})-1]}{(p, r, f, m, s) \stackrel{i}{\rightarrow}\left(p^{\prime}, r^{\prime}, f, m, s\right)} \\
& \vdash \text { writeAllowed }\left(p, r\left(r_{\mathrm{d}}\right)\right) \\
& \frac{m^{\prime}=m\left[r\left(r_{\mathrm{d}}\right) \mapsto r\left(r_{\mathrm{s}}\right)\right]}{(p, r, f, m, s) \stackrel{i}{\rightarrow}\left(p+1, r, f, m^{\prime}, s\right)} \\
& m(p)=\left(\operatorname{cmp} r_{1} r_{2}\right) \\
& s \vdash \operatorname{int} \operatorname{Jump}(p, p+1) \\
& f^{\prime}=f\left[\mathrm{ZF} \mapsto\left(r\left(\mathrm{r}_{1}\right)==r\left(\mathrm{r}_{2}\right)\right)\right. \text {; } \\
& \left.\mathrm{SF} \mapsto\left(r\left(r_{1}\right)<r\left(r_{2}\right)\right)\right] \\
& \text { (Eval-sub) } \\
& m(p)=\left(\operatorname{sub} r_{\mathrm{d}} \mathbf{r}_{\mathbf{s}}\right) \\
& s \vdash \operatorname{intJump}(p, p+1) \\
& f^{\prime}=f[\mathrm{ZF} \mapsto(v==0) \text {; } \\
& \left.\mathrm{SF} \mapsto\left(r\left(\mathrm{r}_{\mathrm{d}}\right)-r\left(\mathrm{r}_{\mathrm{s}}\right)<0\right)\right] \\
& (p, r, f, m, s) \stackrel{i}{\rightarrow}\left(p+1, r^{\prime}, f^{\prime}, m, s\right) \\
& \begin{array}{c}
m(p)=\left(\begin{array}{ll}
j 1 r_{\mathrm{i}}
\end{array} \quad f(\mathrm{SF})==0\right. \\
s \vdash \text { int Jump }(p, p+1) \\
\hline(p, r, f, m, s) \stackrel{i}{\rightarrow}(p+\underset{(\text { Eval-halt })}{1, r, f, m, s)}
\end{array} \\
& (p)=(\text { halt }) \\
& (p, r, f, m, s) \stackrel{i}{\rightarrow}(-1, r, f, m, s)
\end{aligned}
$$

Fig. 8: Operational semantics of $\mathcal{A}+\mathrm{l}$ instructions for programs in the protected memory partition.

guished by any third program interacting with them [Plotkin 1977]. This notion relies on the concept of contexts, which is introduced before presenting the equivalence itself.

Since we consider $\mathcal{A}+$ l programs $P$ that are placed in protected memory and interact with arbitrary unprotected code, contexts model that unprotected code. Thus for any descriptor $s$, contexts $\mathbb{M}$ are partial memories: $\mathbb{M}=m$, where all addresses of $\mathbb{M}$ are unprotected. Formally, given $s, \forall a \in \operatorname{dom}(\mathbb{M}), s \vdash \operatorname{unprotected}(a)$. A program $P$ and a context $\mathbb{M}$ are compatible, denoted as $P \frown \mathbb{M}$, if the memories of $P$ and $\mathbb{M}$ have disjoint domains. Let $\operatorname{dom}(\mathbb{M})=\operatorname{dom}(m)$ if $\mathbb{M}=m$; formally, $P \frown \mathbb{M}$ if $P=\left(m^{\prime}, s\right)$ and $\operatorname{dom}\left(m^{\prime}\right) \cap$ 


$$
\begin{aligned}
& \text { (Eval-protected) (Eval-unprotected) } \\
& \frac{\lfloor\Omega\rfloor \stackrel{i}{\rightarrow}\left\lfloor\Omega^{\prime}\right\rfloor}{\Omega \rightarrow \Omega^{\prime}} \quad \frac{\lceil\Omega\rceil \stackrel{e}{\rightarrow}\left\lceil\Omega^{\prime}\right\rceil}{\Omega \rightarrow \Omega^{\prime}} \\
& \text { (Eval-movl-out) } \\
& m(p)=\left(\operatorname{movl} r_{\mathrm{d}} \boldsymbol{r}_{\mathrm{s}}\right) \\
& s \vdash \operatorname{int} \operatorname{Jump}(p, p+1) \\
& s \vdash \operatorname{readAllowed}\left(p, r\left(r_{\mathrm{s}}\right)\right) \\
& s \vdash \text { unprotected }\left(r\left(r_{\mathrm{s}}\right)\right) \\
& \frac{r^{\prime}=r\left[r_{\mathrm{d}} \mapsto m\left(r\left(r_{\mathrm{s}}\right)\right)\right]}{(p, r, f, m, s) \rightarrow\left(p+1, r^{\prime}, f, m, s\right)} \\
& \text { (Eval-callback (and return)) } \\
& m(p)=\left(j m p r_{\mathrm{d}}\right) \\
& \text { (Eval-movs-out) } \\
& m(p)=\left(\text { movs } \mathrm{r}_{\mathrm{d}} \mathrm{r}_{\mathrm{s}}\right) \\
& s \vdash \operatorname{int} \operatorname{Jump}(p, p+1) \\
& s \vdash \operatorname{writeAllowed}\left(p, r\left(r_{\mathrm{d}}\right)\right) \\
& s \vdash \text { unprotected }\left(r\left(r_{\mathrm{d}}\right)\right) \\
& m^{\prime}=m\left[r\left(\mathrm{r}_{\mathrm{d}}\right) \mapsto r\left(\mathrm{r}_{\mathrm{s}}\right)\right] \\
& (p, r, f, m, s) \rightarrow\left(p+1, r, f, m^{\prime}, s\right) \\
& \text { (Eval-call (and returnback)) } \\
& m(p)=\left(j m p r_{\mathrm{d}}\right) \\
& \frac{p^{\prime}=r\left(r_{\mathrm{d}}\right) \quad s \vdash \operatorname{exitJump}\left(p, p^{\prime}\right)}{(p, r, f, m, s) \rightarrow\left(p^{\prime}, r, f, m, s\right)} \quad \frac{p^{\prime}=r\left(r_{\mathrm{d}}\right) \quad s \vdash \operatorname{entryJump}\left(p, p^{\prime}\right)}{(p, r, f, m, s) \rightarrow\left(p^{\prime}, r, f, m, s\right)}
\end{aligned}
$$

Fig. 9: Operational semantics of whole $\mathcal{A}+$ l programs.

$\operatorname{dom}(\mathbb{M})=\emptyset$. If $P$ and $\mathbb{M}$ are compatible, $\mathbb{M}$ can be plugged with $P$ in order to model interaction between $P$ and $\mathbb{M}$. Formally, if $P \frown \mathbb{M}$ then $\mathbb{M}\left[\left(m^{\prime}, s\right)\right]=\left(m^{\prime}+m, s\right)$.

Programs $P_{1}$ and $P_{2}$ are contextually equivalent, denoted as $P_{1} \simeq P_{2}$, when, for all contexts they interact with, $P_{1}$ diverges if and only if $P_{2}$ also diverges.

Definition 4.4 (Contextual equivalence). $P_{1} \simeq P_{2}$ if $\forall \mathbb{M} . P_{1} \frown \mathbb{M} \wedge \mathbb{M}\left[P_{1}\right] \Uparrow \Longleftrightarrow$ $P_{2} \frown \mathbb{M} \wedge \mathbb{M}\left[P_{2}\right] \Uparrow$.

An implication of this definition is that for $P_{1}$ and $P_{2}$ to be contextually equivalent they must have the same memory descriptor. For the sake of simplicity we will always assume the compatibility of a program and the context it is plugged in, shortening the above definition to: $P_{1} \simeq P_{2}$ if $\forall \mathbb{M} . \mathbb{M}\left[P_{1}\right] \Uparrow \Longleftrightarrow \mathbb{M}\left[P_{2}\right] \Uparrow$.

4.1.3. Trace Semantics. As for the operational semantics, a notion of execution states is required for the trace semantics as well. Execution states, denoted as $\Theta$, are the same as $\Omega$ except that $\Theta$ do not deal with the whole memory but just with its protected partition. So, the memory $m$ of $(p, r, f, m, s)$ spans only the protected memory partition indicated by $s$. Additionally, $\Theta$ can be (unknown, $m, s$ ), an unknown state that models when code is executing in unprotected memory [Jeffrey and Rathke 2005b].

Definition 4.5 (Initial state for traces). The initial state for traces of a program $(m, s)$, denoted as $\Theta_{0}(m, s)$, is the state (unknown, $\left.m, s\right)$.

Below are the labels exhibited by the traces semantics.

$$
\Lambda::=\alpha\left|\tau_{i} \quad \alpha::=\sqrt{ }\right| \gamma ?|\gamma ! \quad \gamma::=\operatorname{call} p(r)| \operatorname{ret} p r_{0}
$$

A label $\Lambda$ can be either an observable action $\alpha$ or a non-observable action $\tau_{i}$. Action $\tau_{i}$ indicates the unobservable action occurred in protected memory. Decorations ? and ! indicate the direction of the observable action: from unprotected to protected code (?) or vice-versa (!). Observable actions include a tick $\sqrt{ }$ indicating that the execution has terminated. Additionally, observable actions are function calls or returns to a certain address $p$, combined with the registers $r$ and flags $f$. Registers and flags are in the labels as they convey information on the behaviour of programs. There are no labels for reads or writes to unprotected memory as those instructions are never generated by the compiler. 
Fig. 10 presents the rules defining the relation $\Theta \stackrel{\bar{\alpha}}{\Longrightarrow} \Theta^{\prime}$, which describe when a state $\Theta$ generates trace $\bar{\alpha}$ and results in state $\Theta^{\prime}$. The traces of a $\mathcal{A}+1$ program $P$ is

$$
\begin{aligned}
& \text { (Trace-internal) } \\
& (p, r, f, m, s) \stackrel{i}{\rightarrow}\left(p^{\prime}, r^{\prime}, f^{\prime}, m^{\prime}, s\right) \\
& s \vdash \operatorname{int} \operatorname{Jump}\left(p, p^{\prime}\right) \\
& (p, r, f, m, s) \stackrel{\tau_{i}}{\longrightarrow}\left(p^{\prime}, r^{\prime}, f^{\prime}, m^{\prime}, s\right) \\
& \text { (Trace-call) } \\
& s \vdash \text { entryPoint }(p) \\
& \text { (unknown, } m, s) \stackrel{\text { call } p(r) ?}{\longrightarrow}(p, r, f, m, s) \\
& \text { (Trace-callback) } \\
& s \vdash \operatorname{exitJump}\left(p, p^{\prime}\right) \\
& \frac{m(p)=\left(\mathrm{jmp} p^{\prime}\right) \quad \mathrm{r}_{4} \neq 0}{\left.(p, r, f, m, s) \stackrel{\text { call } p^{\prime}(r) !}{\longrightarrow} \text { (unknown, } m, s\right)}
\end{aligned}
$$

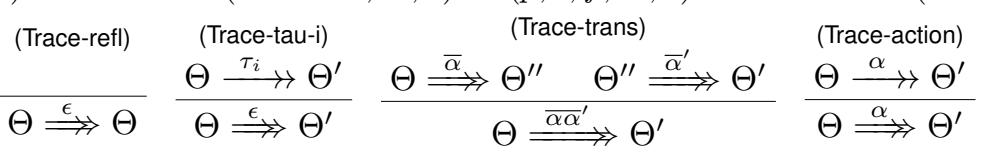

Fig. 10: Rules of the trace semantics of $\mathcal{A}+$.

defined as follows: $\operatorname{Traces}(P)=\left\{\bar{\alpha} \mid \exists \Theta^{\prime} . \Theta_{0}(P) \stackrel{\bar{\alpha}}{\Longrightarrow} \Theta^{\prime}\right\}$. Two programs $P_{1}$ and $P_{2}$ are trace-equivalent, denoted as $P_{1} \simeq_{\mathrm{T}} P_{2}$, if their traces are the same.

Definition 4.6 (Trace equivalence). $P_{1} \simeq_{\mathrm{T}} P_{2}$ if $\operatorname{Traces}\left(P_{1}\right)=\operatorname{Traces}\left(P_{2}\right)$.

An important result of this trace semantics is that when it is applied to compiled components, traces capture all possible behaviours of a compiled component. Thus the trace semantics captures precisely the same notion as the operational semantics. The formal statement of this property is captured by Proposition 4.7 below.

Proposition 4.7 (TRACE SEMANTICS IS EQUIVALENT TO OPERATIONAL SEMANTICS). For any two $\mathcal{A}+$ components $C_{1}^{\downarrow}$ and $C_{2}^{\downarrow}$ obtained from compiling $\mathcal{J}+\mathrm{E}$ components $C_{1}$ and $C_{2}$ with the compilation scheme of Section 3 , we have that: $C_{1}^{\downarrow} \simeq_{\mathrm{T}} C_{2}^{\downarrow} \Longleftrightarrow C_{1}^{\downarrow} \simeq C_{2}^{\downarrow}$.

Proposition 4.7, whose proof is detailed in [Patrignani and Clarke 2014], drives the proof strategy for the main result, which is presented in Section 5. That work considers an assembly language enhanced with a protected modules architecture, yet that formalisation differs slightly from the one presented in this paper. In that work, the language has two stacks and switching between them is done by using call and ret instructions. In this work, only one stack is present and jmp instructions are used to switch between memory partitions. Intuitively, that formalisation has a built-in notion of secure and insecure stacks, while this work only has an insecure one and the secure stack is set up by the secure compiler. The results of Proposition 4.7 can thus be applied in this paper as well.

\subsection{Formalisation of $\mathcal{J}+\mathrm{E}$}

This section presents the formalisation of the dynamic semantics of $\mathcal{J}+\mathrm{E}$, which borrows extensively from that of Java Jr. [Jeffrey and Rathke 2005b]. Its syntax was already presented in Fig. 2 in Section 2.3. 
4.2.1. Dynamic Semantics. The dynamic semantics is given in terms of a relation $(C ; S \vdash E) \rightarrow\left(C^{\prime} ; S^{\prime} \vdash E^{\prime}\right)$ that models the evolution of component $C$ executing expression $E$ with stack $S$ to $C^{\prime}$ executing $E^{\prime}$ with stack $S^{\prime}$. A binding $B$ is a list of associations from variables to values, $B::=\emptyset \mid B ;(x \mapsto v)$. The lookup of the value associated to a variable, denoted as $B(x)$, returns $v$ if $(x \mapsto v) \in B$ and is undefined otherwise. A stack $S$ is a list of bindings $S::=\bar{B}$, lookup and addition are always made to the top of the stack, so if $S=B_{1}, \cdots, B_{n}$, then $S(x)$ stands for $B_{1}(x)$ and $S,(x \mapsto v)$ stands for $B_{1},(x \mapsto v)$. The expression being executed is immersed in an evaluation context $\mathbb{E}$, which models the environment in which the evaluation takes place. The syntax of evaluation contexts is:

$$
\begin{aligned}
\mathbb{E}::= & {[\cdot]|\mathbb{E} . m(\bar{E})| \text { p.o.m }(\bar{v}, \mathbb{E}, \bar{E})|\mathbb{E} . f| \mathbb{E} . f=E \mid \text { v.f }=\mathbb{E} \mid \text { new } t(\bar{v}, \mathbb{E}, \bar{E}) } \\
& \mid \mathbf{i f}(\mathbb{E})\left\{E_{T}\right\} \text { else }\left\{E_{F}\right\}|\mathbb{E} ; E| \mathbb{E} \text { in } p|\operatorname{var} x: t=\mathbb{E}| \text { return } \mathbb{E} \\
& \mid \mathbb{E} \text { op } E \mid v \text { op } \mathbb{E}|\operatorname{try} \mathbb{E} \operatorname{catch}(x: t) E| \text { throw } \mathbb{E} \mid \text { exit } \mathbb{E}
\end{aligned}
$$

Rules for reductions of the form $(C ; S \vdash E) \rightarrow\left(C^{\prime} ; S^{\prime} \vdash E^{\prime}\right)$ are presented in Fig. 11 and 12. Contextual equivalence for $\mathcal{J}+E$ programs is defined based on $\mathcal{J}+E$ contexts $\mathbb{C}$, which are components with a hole, denoted with $\mathbb{C}[\cdot]$ [Jeffrey and Rathke 2005b]. The hole can be filled with another component $C$ to denote the interaction between $C$ and the context. Assume the context defines a Main package with a Main class and a main method that identify where the execution starts. We overload the $\simeq$ notation and define contextual equivalence for $\mathcal{J}+\mathrm{E}$ programs as follows: $C_{1} \simeq C_{2}$ if $\forall \mathbb{C}$. $\mathbb{C}\left[C_{1}\right] \Uparrow \Longleftrightarrow \mathbb{C}\left[C_{2}\right] \Uparrow$ knowing that this relation will not be mistaken for the $\mathcal{A}+\mathrm{l}$ one.

\section{FULL ABSTRACTION OF THE COMPILATION OF $\mathcal{J}+$ E TO $\mathcal{A}+1$}

This section firstly presents the algorithm mentioned in Section 2.4 through a series of examples (Section 5.1). Then it presents the proof of full abstraction of the compilation scheme of Section 3, which relies on the algorithm (Section 5.2).

\subsection{The Algorithm}

This section presents the algorithm which takes as input two different, low-level traces $\bar{\alpha}_{1}$ and $\bar{\alpha}_{2}$ and two components $C_{1}$ and $C_{2}$ and outputs a high-level component $C$ that differentiates $C_{1}$ and $C_{2}$. Traces $\bar{\alpha}_{1}$ and $\bar{\alpha}_{2}$ were generated by $C_{1}^{\downarrow}$ and $C_{2}^{\downarrow}$ when interacting with the same, unknown external memory. This section presents several examples of the expected output of the algorithm when different traces and components are input. The examples illustrate crucial cases the algorithm needs to consider when creating the output component.

In the following, the adjective internal denotes objects (classes) that are allocated (defined) by components $C_{1}$ and $C_{2}$. The adjective external denotes objects (classes) that are allocated (defined) by the output component.

General idea. The algorithm analyses actions in the low-level traces $\bar{\alpha}_{1}$ and $\bar{\alpha}_{2}$. Those actions can be: call, return, callback, returnback and termination $(\sqrt{ })$. Actions that appear at even-numbered positions in a trace are calls or returnbacks, generated from the external memory. Actions that appear at odd-numbered positions are returns or callbacks, generated by $C_{1}$ or $C_{2}$. This partitioning is because execution starts in unprotected memory.

For the algorithm to be correct, it must detect when two different actions are encountered at an odd position in a trace. Assuming the first different actions are at index $i$, the algorithm produces code that replicates the first $i-1$ actions. Then, it produces code that, based on the difference in the $i$-th action, either diverges or terminates based on which component it is interacting with. 


$$
\begin{aligned}
& \text { (Eval-method) } \\
& C . v=\{\text { package } p \text {; object } v: t \text { implements } \bar{t}\{\bar{F}\}\} \\
& \text { public } m(\bar{x}: \bar{t}): t^{\prime}\{\text { return } E ;\} \in C . t . m \text { ths } \\
& (C ; S \vdash \mathbb{E}[v \cdot m(\bar{v})]) \rightarrow(C ; \emptyset, S \vdash \mathbb{E}[\text { return } E[v / \text { this, } \bar{v} / \bar{x}] \text { in } p]) \\
& \text { (Eval-return) } \\
& (C ; B, S \vdash \mathbb{E}[\text { return } v]) \rightarrow(C ; S \vdash \mathbb{E}[v]) \\
& \text { (Eval-field) } \\
& C . v=\{\text { package } p \text {; object } v: t \text { implements } \bar{t}\{\bar{F}\}\} \quad f=u \in \bar{F} \\
& (C ; S \vdash \mathbb{E}[v . f]) \rightarrow(C ; S \vdash \mathbb{E}[u]) \\
& \text { (Eval-field-update) } \\
& C . v=\{\text { package } p \text {; object } v: t \text { implements } \bar{t}\{\bar{F}\}\} \quad(f=u ;) \in \bar{F} \\
& C^{\prime}=C+\left\{\text { package } p \text {; object } v: t \text { implements } \bar{t}\left\{\bar{F}^{\prime}\right\}\right\} \quad \bar{F}^{\prime}=\bar{F}+(f=u) \\
& (C ; S \vdash \mathbb{E}[v . f=u]) \rightarrow\left(C^{\prime} ; S \vdash \mathbb{E}[u]\right) \\
& \text { (Eval-new) } \\
& \text { C.p.c.flds }=\bar{f}: \bar{t} \quad \text { p.o } \notin \operatorname{dom}(C) \\
& C^{\prime}=C+\{\text { package } p \text {; object } o: p \text {.c implements } \epsilon\{\bar{f}=\bar{v}\}\} \\
& (C ; S \vdash \mathbb{E}[\text { new p.c }(\bar{v})]) \rightarrow\left(C^{\prime} ; S \vdash \mathbb{E}[\text { p.o }]\right) \\
& \text { (Eval-if-true) } \\
& \frac{v=\text { true }}{\left(C ; S \vdash \mathbb{E}\left[\mathbf{i f}(v)\left\{E_{T}\right\} \text { else }\left\{E_{F}\right\}\right]\right) \rightarrow\left(C ; S \vdash \mathbb{E}\left[E_{T}\right]\right)} \\
& \text { (Eval-if-false) (Eval-coercion) } \\
& \frac{v=\text { false }}{\left(C ; S \vdash \mathbb{E}\left[\mathbf{i f}(v)\left\{E_{T}\right\} \text { else }\left\{E_{F}\right\}\right]\right) \rightarrow\left(C ; S \vdash \mathbb{E}\left[E_{F}\right]\right)} \stackrel{(C ; S \vdash \mathbb{E}[v \text { in } p]) \rightarrow(C ; S \vdash \mathbb{E}[v])}{\text { (Eval-local-var) }}
\end{aligned}
$$

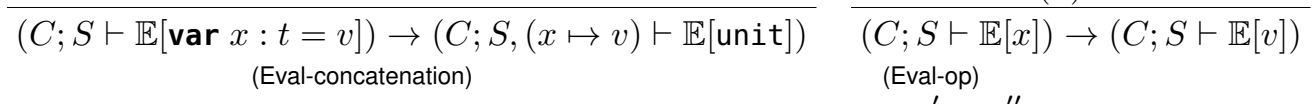

$$
\begin{aligned}
& \frac{(C ; S \vdash \mathbb{E}[v ; E]) \rightarrow(C ; S \vdash \mathbb{E}[E])}{\text { (Eval-try) }} \frac{v \text { op } v^{\prime}=v^{\prime \prime}}{\left(C ; S \vdash \mathbb{E}\left[v \text { op } v^{\prime}\right]\right) \rightarrow\left(C ; S \vdash \mathbb{E}\left[v^{\prime \prime}\right]\right)} \\
& v \neq \text { throw } v^{\prime} \\
& (C ; S \vdash \mathbb{E}[\operatorname{try}\{v\} \operatorname{catch}(x: t)\{E\}]) \rightarrow(C ; S \vdash \mathbb{E}[v]) \\
& \text { (Eval-catch) } \\
& \frac{C . v=\{\text { package } p \text {; object } v: t \text { implements } \bar{t}\{\bar{F}\}\} \quad t<: t^{\prime}}{\left(C ; S \vdash \mathbb{E}\left[\operatorname{try}\{\text { throw } v\} \operatorname{catch}\left(x: t^{\prime}\right)\{E\}\right]\right) \rightarrow(C ; S \vdash \mathbb{E}[E[v / x]])} \\
& \text { (Eval-catch-fail) } \\
& \frac{C . v=\{\text { package } p ; \text { object } v: t \text { implements } \bar{t}\{\bar{F}\}\} \quad t<t t^{\prime}}{\left(C ; S \vdash \mathbb{E}\left[\operatorname{try}\{\text { throw } v\} \operatorname{catch}\left(x: t^{\prime}\right)\{E\}\right]\right) \rightarrow(C ; S \vdash \mathbb{E}[\operatorname{throw} v])} \\
& \text { (Eval-exit) } \\
& \overline{(C ; S \vdash \mathbb{E}[\operatorname{exit} v]) \rightarrow(C ; S \vdash v)}
\end{aligned}
$$

Fig. 11: Dynamic semantics of $\mathcal{J}+\mathrm{E}$. The subtyping relation is denoted by $<$ :

The algorithm has been implemented in Scala, and it outputs Java components that adhere to the $\mathcal{J}+\mathrm{E}$ formalisation. ${ }^{5}$ For implementation purposes, instead of diverging

\footnotetext{
${ }^{5}$ Available at http://people.cs.kuleuven.be/ marco.patrignani/Publications.html.

ACM Transactions on Programming Languages and Systems, Vol. ?, No. ?, Article ??, Publication date: January ????.
} 


$$
\begin{gathered}
\text { (Eval-throw-sequence) } \\
\frac{(C ; S \vdash \mathbb{E}[\text { throw } v ; E]) \rightarrow(C ; S \vdash \mathbb{E}[\text { throw } v])}{(\text { Eval-throw-throw) }} \\
\frac{(C ; S \vdash \mathbb{E}[\text { throw throw } v]) \rightarrow(C ; S \vdash \mathbb{E}[\text { throw } v])}{(\text { Eval-throw-var) }} \\
\frac{(C ; S \vdash \mathbb{E}[\operatorname{var} x: t=\text { throw } v]) \rightarrow(C ; S,(x \mapsto \text { throw } v) \vdash \mathbb{E}[\text { throw } v])}{(\text { Eval-throw-new) }} \\
\frac{(E ; S \vdash \mathbb{E}[\text { new throw } v]) \rightarrow(C ; S \vdash \mathbb{E}[\text { throw } v])}{(\text { Eval-throw-if) }} \\
\hline\left(C ; S \vdash \mathbb{E}\left[\mathbf{i f}(\text { throw } v)\left\{E_{T}\right\} \text { el se }\left\{E_{F}\right\}\right]\right) \rightarrow(C ; S \vdash \mathbb{E}[\text { throw } v])
\end{gathered}
$$

Fig. 12: Dynamic semantics of $\mathcal{J}+\mathrm{E}$, rules for exception propagation.

in a case and terminating in the other the implementation terminates with value 1 or 2 . This formulation of contextual equivalence is equivalent to ours, yet more amenable to an implementation [Curien 2007].

Starting point. The algorithm starts by creating a knowledge base about $C_{1}$ and $\mathrm{C}_{2}$. The knowledge base contains all signatures of internally- and externally-defined methods, as well as high- and low-level identities of static objects and externs. This is because the algorithm needs to be able to differentiate, for example, whether a type is internally or externally defined, or what are the identities of static objects. Then, a code skeleton for the output component is created, based on the structure of the distinguished import package (DIP) of $C_{1}$ and $C_{2}$.

For all interfaces $i$ defined in the DIP, a class $i_{-}$c is created. An object staticFor $i$ of type $i_{-} \mathrm{c}$ is then created. Classes $i_{-} \mathrm{c}$ contain dummy implementations of all methods defined in $i$ and in all interfaces $i$ extends. These method implementations return a value whose type matches the expected return type: 0 for type Int, unit for type Unit and null otherwise. A method called defaultcreate() is added to all classes $i_{-} \mathrm{c}$, it is implemented as follows:

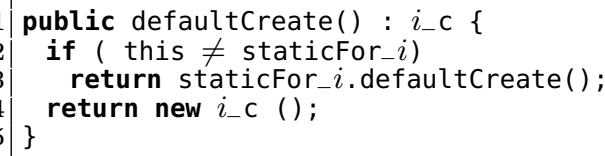

Methods defauttcreate( ) are responsible for allocating external objects, they will be called only on objects staticFor_i. Constructors inside defaultCreate() are supplied standard values for their parameters: 0 for type Int, false for type Bool, unit for type Unit and null otherwise. These classes have no fields, as they are never accessed by protected code. For the sake of simplicity, the following examples have constructors with no parameters.

The output component is extended with extra classes. Firstly, class Tester containing the main method is added; it is required for the execution to start. Other needed classes will be introduced and motivated by the following examples. 
Code examples. The following examples present different implementations of $C_{1}$, on the left, and of $C_{2}$, on the right. Components $C_{1}$ and $C_{2}$ are modifications of the code in Listing 1 , whose DIP is defined in Listing 3 below. Omitted code is the same in both $C_{1}$

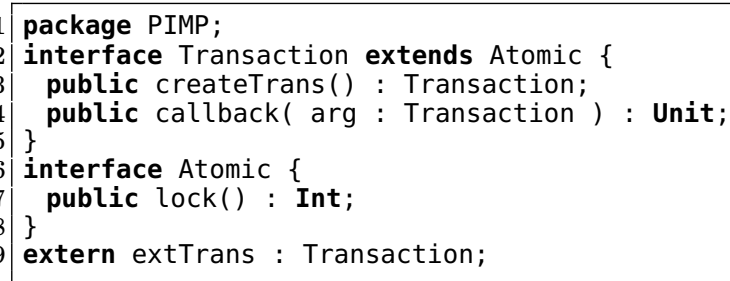

Listing 3: Example of a distinguished import package.

and $C_{2}$ and can be found in Listing 1 . Each code fragment is followed by the low-level trace it generates: $\overline{\alpha_{1}}$ and $\overline{\alpha_{2}}$ for $C_{1}$ and $C_{2}$ respectively. The examples also describe what the algorithm must do in order to create the correct output before presenting the output produced for each case.

The low-level traces will be massaged to aid understanding. For example, given that object extAccount is compiled to identity $0 \times 123$ and that the entry point of method createAccount is located at address $0 \times 456$, the low-level label call $0 \times 456\left(r\left[r_{4}=0 \times 123\right]\right)$ is written as extAccount. createAccount (). This abstraction is safe, as it does not introduce additional information, it merely massages the present one into a more humanreadable form. Numbers in italic font, e.g. 1 , refer to indexes from $\mathcal{O}$, while identities of externally allocated objects are numbers in hexadecimal form.

Example 5.1 (Different returned values). Consider the following implementations for $C_{1}$ and $C_{2}$.

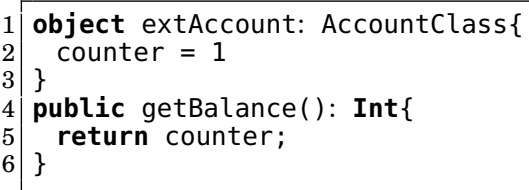

Trace $\overline{\alpha_{1}}$ for $C_{1}$ is extAccount.getBalance()?·ret 1!·extAccount.getBalance()?·ret 1!, while trace $\overline{\alpha_{2}}$ for $C_{2}$ is extAccount.getBalance()? · ret 1! - extAccount.getBalance()? · ret 2!. In this example, the produced code needs to differentiate between $C_{1}$ and $C_{2}$ based on the type of expected returned values. These types can be either: primitive, internal, external. With primitive-typed values the differentiation is based on the different values returned by $C_{1}$ or $C_{2}$, in this case 1 and 2 respectively.

This example highlights how both the algorithm and the produced code need to keep track of the index of the action they replicate. To that end, the algorithm maintains a global variable. The produced code is extended with a class Helper and a static object oc implementing it. Helper contains a field step with methods getStep() and incrementStep ( ), the latter increases the value of step by one. Additionally, it contains a method diverge( ) that recursively calls itself, which is used to achieve divergence. As oc is static, its fields are global variables for the output component.

The algorithm outputs the code of Listing 4 . The first actions generate the code in lines 2 to 6 , thus it is wrapped in an if-statement that makes the generated code take place only when the considered action is the first: i.e. step is 0 . The second actions are 


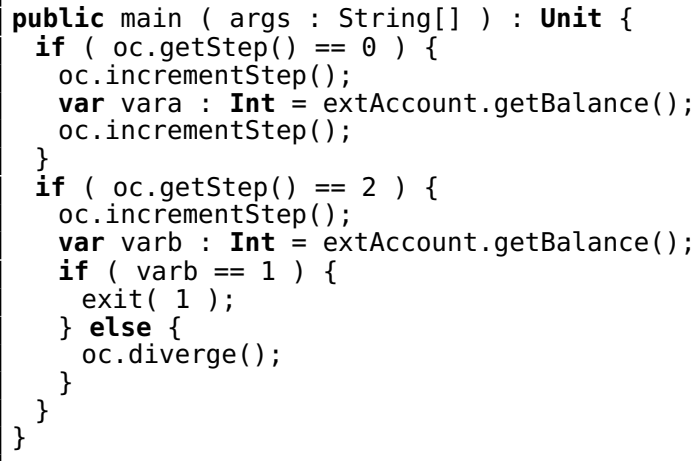

Listing 4: Output of the algorithm for Example 5.1

responsible for incrementing step in line 5 . The third actions generate the code in lines 7 to 11, while the fourth actions, the different ones, generate the code in line 10 .

The approach of this example is similar to what the algorithm does in case the difference in the traces is in primitive-typed parameters of a callback. In that case, instead of creating fresh variable varb, the produced code performs the differentiation by using the name of the parameter which has the different value.

\section{Example 5.2 (Different internally-typed returned object).}

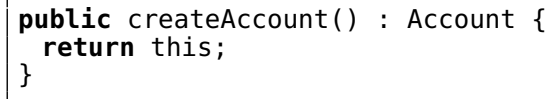

Trace $\overline{\alpha_{1}}$ is extAccount.createAccount()? - ret extAccount!, while trace $\overline{\alpha_{2}}$ is extAccount.createAccount ()? - ret 1 !. In this case the produced code must be able to differentiate between two return values that are internal objects. They are given different indexes in $\mathcal{O}$. Here, $C_{1}$ returns a known object: extAccount, while $C_{2}$ returns a new object: index 1 in $\mathcal{O}$.

To achieve differentiation in this case, the produced code needs to keep track of internally allocated objects. For this it relies on a list internals provided by oc. In order for internal objects to be accessible, they are wrapped with a new class: Internal that has two fields. The first, of type $0 \mathrm{bj}$, contains a reference to an internal object. The second, name, can be used to filter the search for objects. No two objects with the same name can be added to internals, which is initialised with entries for all known static objects. Elements of this list can be accessed via method getNameBy0bject ( o ), which returns the name of object o or null if o is not in internals. The algorithm has a table with the low-level identities of all dynamically-allocated objects in order to generate correct code when retrieving internals as in line 6 in the code below.

The algorithm outputs the code of Listing 5 . Line 5 has no effect, since internals already has an entry for extAccount. In case $C_{1}$ and $C_{2}$ were swapped, line 5 would bind $\mathrm{f}$ to name 1 , ensuring the execution of the else-branch in the if statement in line 6.

This example scales to different internally-typed parameters in a callback.

Example 5.3 (Different method of a callback). 


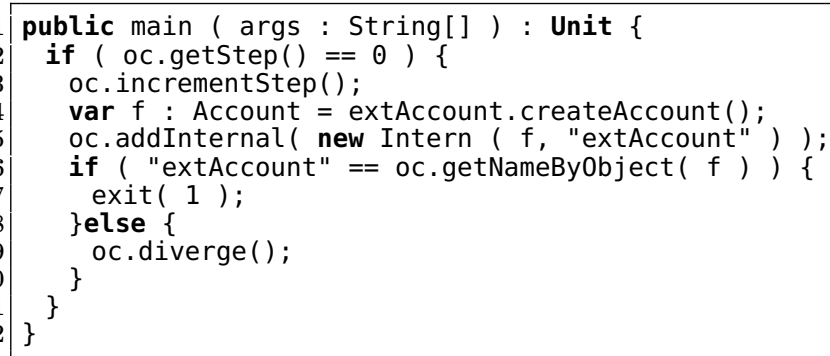

Listing 5: Output of the algorithm for Example 5.2

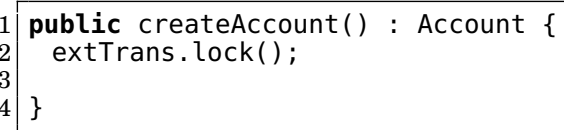

Trace $\overline{\alpha_{1}}$ is extAccount.createAccount()? - extTrans.lock()!, while trace $\overline{\alpha_{2}}$ is extAccount.createAccount ()? extTrans.createTransaction()!. In this example, $C_{1}$ performs a callback to method lock, while $C_{2}$ performs it to method createTransaction.

To achieve differentiation in this case, the algorithm needs to keep track of the current method, since it indicates where the differentiating code will be placed. The current method is recorded in a stack which is initially set to method main in class Tester. Callbacks indicate that the current method is changed to a new entry, returnbacks indicate that the current method is restored to a previous one. Thus, whenever a callback to method $m$ of class $c$ is performed, an entry of the form c.m is pushed on the stack. A returnback pops the head of the current method stack.

The algorithm outputs the code of Listing 6 . Notice that the if-statements of lines 8

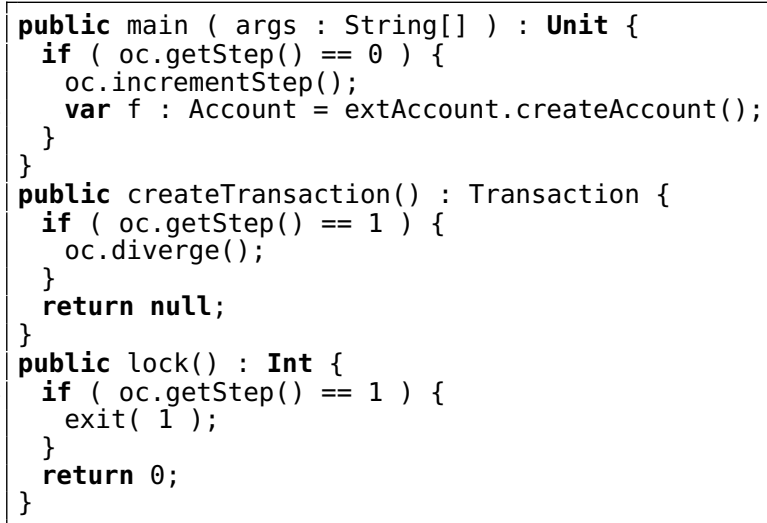

Listing 6: Output of the algorithm for Example 5.3

and 12, whose addition was discussed in Example 5.1, help the produced code achieve differentiation in this case as well. Should methods createTransaction() or lock() be called multiple times, the if-guard ensures that the differentiation only happens at the right time. 
Example 5.4 (Different callee of a callback).
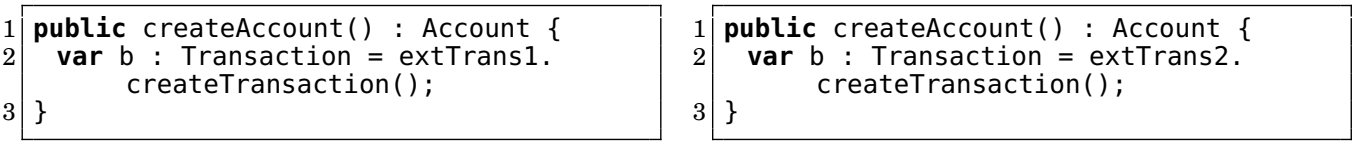

Trace $\overline{\alpha_{1}}$ is extAccount.createAccount()? - extTrans 1.createTransaction()!, while trace $\overline{\alpha_{2}}$ is extAccount.createAccount ()? · extTrans2.createTransaction()!. In this case the difference is the external object on which the second callback is performed. Here, $C_{1}$ calls createTransaction() on extTrans1, while $C_{2}$ calls it on extTrans 2 .

In order to achieve this differentiation, the produced code needs to keep track of external objects similarly to how it needed to keep track of internal objects in Example 5.2. All external objects must be bound to a name, just as the internally allocated ones are. For this purpose, a class Listable is created, all the classes i_c extend Listable. Listable contains a name and a type field, with getters and setters. It also contains a method setAndRegister $(n, t)$, that sets name $=n$, type $=t$ and adds the object to a list of Listable called externals that is kept in object oc. Object oc contains method getExternal $(n, t)$ to retrieve these objects based on name and type.

The algorithm outputs the code of Listing 7 . Fields name and type for external static

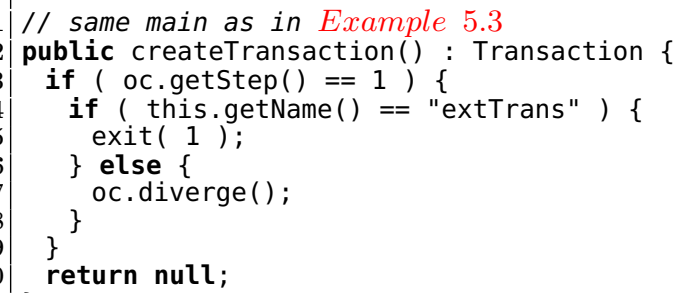

Listing 7: Output of the algorithm for Example 5.4

objects are initialised in the first instructions of the main. That code is omitted for brevity.

Example 5.5 (Different callee of a callback \#2).

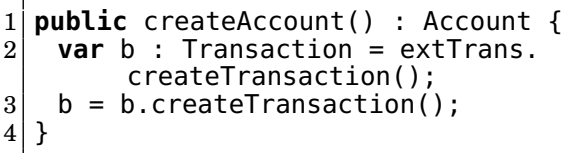

Trace $\overline{\alpha_{1}}$ is extAccount.createAccount()? . extTrans.createTransaction()! . ret $0 \times 6$ ? - $0 \times 6$.createTransaction()! while $\overline{\alpha_{2}}$ is extAccount.createAccount()? . extTrans.createTransaction()! · ret $0 \times 6$ ? · extTrans.createTransaction()!. In this case the difference is the external object on which a callback is performed. Here, $C_{1}$ calls createTransaction() on $0 \times 6$, while $C_{2}$ calls the same method on extTrans.

The algorithm outputs the code of Listing 8. Lines 14 to 17 ensure that if an external object is not found in the list externals, it is allocated by calling to the default factory method and then added to externals. Fields name and type for external static objects are assumed to be initialised in the first instructions of the main. That code is omitted for brevity. 
Casts are not present in $\mathcal{J}+\mathrm{E}$, yet casting an object $o$ to a type $t$ can be modelled by throwing $o$ and catching an exception of type $t$. The cast is presented for the sake of simplicity.

Example 5.7 (Traces with different actions).
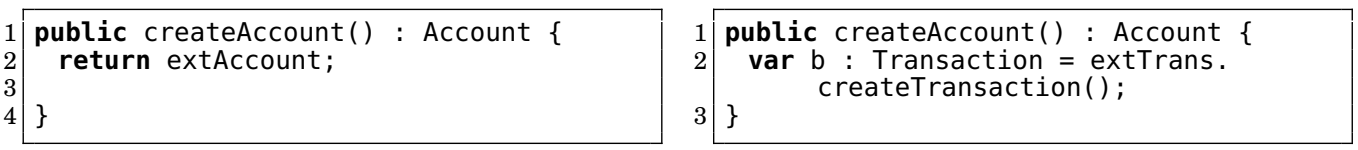

Trace $\alpha_{1}$ is extAccount.createAccount()?. ret 1!, while trace $\alpha_{2}$ is extAccount.createAccount()? $\cdot 0 \times 6$. createTransaction()!. In this case the algorithm needs to identify the two different locations where execution will be after the different actions are executed. The concept of current method introduced in Example 5.3 can be used in this case as well in order to determine where to place the code that performs the differentiation.

The algorithm outputs the code of Listing 10.

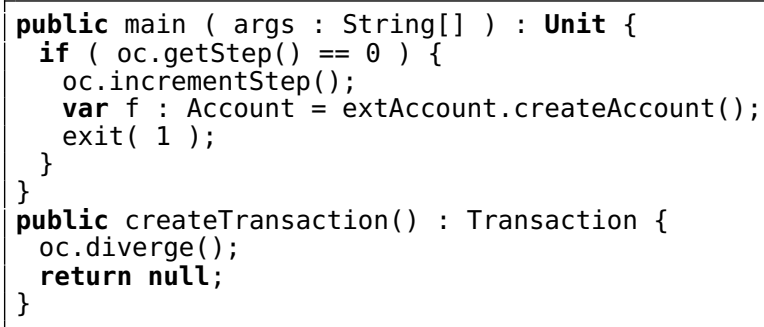

Listing 10: Output of the algorithm for Example 5.7

This examples covers also other cases of different actions, such as a ret and a $\sqrt{ }$ and a call and a $\sqrt{ }$.

Example 5.8 (Traces of different length).

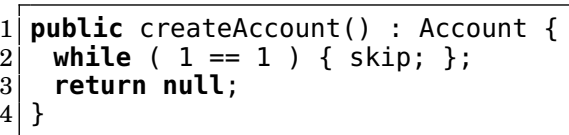

Trace $\alpha_{1}$ is extAccount.createAccount ()?, while trace $\alpha_{2}$ is extAccount. createAccount ()? · ret 1 !.

The algorithm outputs the code of Listing 11 . When control is returned to main after

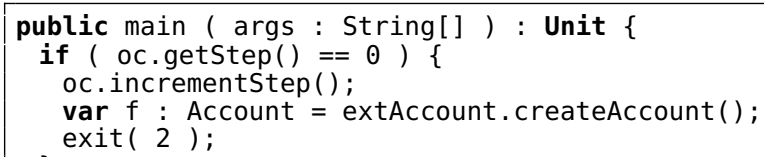

Listing 11: Output of the algorithm for Example 5.8 
a call to createAccount (), it means that the output component is interacting with $C_{2}$. In this case the produced code terminates via the expression of line 5. Divergence is accomplished by $C_{1}$.

As previously mentioned, exceptions are compiled as call and callbacks with a single parameter: the object id of what is being thrown. Whenever a call to the throw entry point is detected in the traces, a throw expression is written in place of a normal method call. The signature of the method must declare that it will throw an exception, otherwise the last action in the trace will be this thrown exception. This means that after this trace the execution will halt, as no exception to be thrown is registered, but this contradicts the presence of a difference in $\overline{\alpha_{1}}$ and $\overline{\alpha_{2}}$. Calls to methods that can throw exceptions are wrapped in a try/catch block. If a jump to the external exception handler is detected, then the code is written in the catch block. Otherwise, if a normal action is detected, the code is written in the try block, below the method call.

\subsection{Full Abstraction of the Compilation Scheme}

ASSUMPTION 1 (COMPILER PRESERVES BEHAVIOUR). The secure compiler is assumed to output target-level programs that behave as the corresponding input program. Thus a source-level expression is translated into a list of target-level instructions that preserve the behaviour. By this, we mean that $C_{1}^{\downarrow} \simeq C_{2}^{\downarrow} \Rightarrow C_{1} \simeq C_{2}$.

Notation. Indicate the $i$-th action of a trace $\bar{\alpha}$ as $\alpha^{(i)}$.

ThEOREM 5.9 (Algorithm CORRECTNESS). For any two source-level components $C_{1}$ and $C_{2}$ that, once compiled, exhibit a different trace semantics, the algorithm of Section 5.1 outputs a component $C$ that differentiates between $C_{1}$ and $C_{2}$ (assuming there is no overflow of the secure stack and of the secure heap). Formally: $C_{1}^{\downarrow} \neq_{\mathrm{T}} C_{2}^{\downarrow} \Rightarrow$ $C_{1} \neq C_{2}$.

Proof. As presented in Section 4.1.3, trace semantics deals with sets of traces, while the algorithm inputs single traces. Moreover, these single traces must be the same up to a !-decorated action. The two different single traces are obtained as follows. Since $C_{1}^{\downarrow} \neq_{\mathrm{T}} C_{2}^{\downarrow}$, we have that $\operatorname{Traces}\left(C_{1}^{\downarrow}\right) \neq \operatorname{Traces}\left(C_{2}^{\downarrow}\right)$, thus there exists a trace $\bar{\alpha}$ that belongs to either only $\operatorname{Traces}\left(C_{1}^{\downarrow}\right)$ or only $\operatorname{Traces}\left(C_{2}^{\downarrow}\right)$ but not to both. Assume wlog that $\bar{\alpha} \in \operatorname{Traces}\left(C_{1}^{\downarrow}\right)$. The trace $\bar{\alpha}$ can be split in two parts $\overline{\alpha_{s}}$ and $\overline{\alpha_{d}}$ such that $\bar{\alpha}=\overline{\alpha_{s} \alpha_{d}}$ and such that $\overline{\alpha_{s}}$ is the longest prefix of all traces of $\overline{\alpha_{2}}$. So, there exists a trace $\overline{\alpha^{\prime}} \in$ $\operatorname{Traces}\left(C_{2}^{\downarrow}\right)$ that can be split in two parts $\overline{\alpha_{s}}$ and $\overline{\alpha_{d}^{\prime}}$ such that $\overline{\alpha^{\prime}}=\overline{\alpha_{s}} \overline{\alpha_{d}^{\prime}}$ and $\overline{\alpha_{d}} \neq \overline{\alpha_{d}^{\prime}}$. Additionally, $\overline{\alpha_{2}}$ must have even length, so that the different action in the traces is !-decorated and thus generated by either $C_{1}$ or $C_{2}$. Trace $\overline{\alpha^{\prime}}$ always exists, it could be an empty trace, it could be composed by an empty $\overline{\alpha_{s}}$ and, possibly, by an empty $\overline{\alpha_{d}^{\prime}}$. The traces input for the algorithm are $\overline{\alpha_{1}}=\overline{\alpha_{s} \alpha_{d}}$ and $\overline{\alpha_{2}}=\overline{\alpha_{s}} \overline{\alpha_{d}^{\prime}}$.

The proof analyses all possible differences in $C_{1}^{\downarrow} \neq_{\mathrm{T}} C_{2}^{\downarrow}$ and proves that the output of the algorithm differentiates between $C_{1}$ and $C_{2}$, so $C_{1} \neq C_{2}$. For each possible difference, the proof refers to an example from Section 5.1 that generate the context capable of performing the differentiation.

- traces of different length (Example 5.8);

- different kind of actions (Example 5.7);

- same kind of actions with differences in their structure:

- return action

— different primitive-typed value (Example 5.1);

— different internally-typed value (Example 5.2); 
— different externally-typed value (Example 5.6);

- call action

- different callee (Example 5.4 and 5.5);

- different primitive-typed parameter (Example 5.1);

- different internally-typed parameter (Example 5.2);

- different externally-typed parameter (Example 5.6);

— different method (Example 5.3).

As those listed above are the only differences that can appear in two different traces, and since they all present a counterexample that reaches the contradiction, the theorem holds.

THEOREM 5.10 (Full ABSTRACTION OF THE COMPIlation SCHEME). For any two source-level components $C_{1}$ and $C_{2}$, we have: $C_{1} \simeq C_{2} \Longleftrightarrow C_{1}^{\downarrow} \simeq C_{2}^{\downarrow}$.

Proof. The equivalence is split into two subgoals. The direction $\Leftarrow$ holds due to Assumption 1. The direction $\Rightarrow$ is reversed to the equivalent statement: $C_{1}^{\downarrow} \neq C_{2}^{\downarrow} \Rightarrow$ $C_{1} \neq C_{2}$. Apply Proposition 4.7 to restate the statement as $C_{1}^{\downarrow} \neq_{\mathrm{T}} C_{2}^{\downarrow} \Rightarrow C_{1} \neq C_{2}$. Apply Theorem 5.9 to prove the statement.

\section{IMPLEMENTATION AND BENCHMARKS}

This section details the architecture adopted to develop the secure compiler of Section 3 (Section 6.1), it compares that architecture to the Intel SGX (Section 6.2) and it presents benchmarking of the overhead introduced by the secure compiler (Section 6.3).

\subsection{Protected Module Architecture}

The secure compilation scheme of Section 3 relies on the target language having a protected module architecture for it to be secure. In order to time the overhead of the secure compiler we implemented it for the Fides architecture [Strackx and Piessens 2012].

The Fides architecture implements precisely the protection mechanism described in Section 2.1 in a very small TCB: $~ 7000$ lines of code. Fides consists of a hypervisor that runs two virtual machines: the secure VM handles the protected memory section and the Legacy VM handles the unprotected one. Switching between the two virtual machines of Fides (i.e. performing calls and callbacks) is more costly than in a hardwarebased implementation. However, we are not interested in this, the overhead that we are interested in timing is the one provided by the additional checks introduced by the secure compiler.

We now give a brief description of the Fides architecture, followed by an informal presentation of the implementation of the secure compiler.

Legacy VM. The Legacy VM executes all legacy applications and other code in unprotected memory. Using virtualisation techniques, this virtual machine is able to execute commodity operating systems and legacy applications without any modification. From the point of view of the Legacy VM, the only difference compared to running on bare hardware is that certain memory locations are inaccessible. More specifically, two memory regions are inaccessible to the Legacy VM: (1) the memory region reserved for the hypervisor and (2) the protected memory region as defined in our low-level machine model. Whenever an access to these memory locations is attempted, execution traps to the hypervisor.

Hypervisor. The hypervisor serves two simple purposes. First, it offers a coarsegrained memory protection: it prevents any code executing in the Legacy VM from 
accessing the protected module or the security measure itself (as discussed above) and it prevents the Secure VM from accessing the hypervisor.

Second, the hypervisor implements a simple scheduling algorithm. When the Legacy VM calls an entry point in the protected module, control goes to the hypervisor who then schedules the Secure VM. Execution control only returns to the Legacy VM when the protected module either returns or performs a callback to unprotected memory.

Secure VM. The Secure VM can access all memory, with the exception of memory containing the hypervisor. The fine-grained memory access control mechanism is implemented by a security kernel running in this VM, as follows. First, when a request is received from the hypervisor to execute a method in the protected module, the requested entry point is checked against a list of valid entry points provided in the module's memory descriptor. When this check passes, the hardware memory management unit is set up to allow memory accesses to the module's memory region and execution proceeds from the entry point that was called. When execution tries to jump back out of the protected module, a page fault is generated, which causes the security kernel to ultimately return execution control to the Legacy VM.

6.1.1. Secure Build Tools. To simplify the development and benchmarking of modules, we developed a fully abstract compilation tool chain using the LLVM compiler and the ELF Tool Chain library. ${ }^{6}$

Compilation of modules is done in two steps. First, for every function that is annotated as an entry point, an entry in the module's entry point table is created. Each entry checks whether an initialisation function needs to be called, sets up the stack pointer and stores the return address in unprotected memory on the stack. After the correct function is called, registers not carrying a result value are cleared. Wrapper functions for each entry point are also generated, in order to simplify the calling of the module. Secondly, the source code is analysed and modified so that every call site that results in a callback to unprotected memory flows through the callback entry point. Registers not carrying a function parameter are reset, so that no information is leaked.

After compilation of the module - possibly resulting in multiple ELF files if the source code was split over multiple files - a secure linker lays out the module in memory according to Fides' requirements. Protected modules must start with the entry table, followed by all compiled code and read-only data such as strings (i.e., the Code section) and the runtime stack and security sensitive variables (i.e., the Data section).

\subsection{Intel Software Guard eXtensions}

In June 2013 Intel publicly disclosed its work on Software Guard eXtensions (SGX) [McKeen et al. 2013; Anati et al. 2013; Hoekstra et al. 2013]. SGX provides a hardware-implemented isolation mechanism that is very similar to Fides [Strackx and Piessens 2012] and related protected module architectures [Strackx et al. 2013; Noorman et al. 2013; Strackx et al. 2010; Avonds et al. 2013]. Enclaves is the SGX terminology for what we called modules in this paper. Enclaves live in the same address space as unprotected parts of the application and can only be accessed through an explicitly exposed interface. Direct memory accesses from unprotected memory to enclaves memory regions are prevented. Enclaves, like modules, have full access to unprotected parts of the application. We believe that the presented fully abstract compilation scheme can be easily ported to SGX-enabled platforms, modulo small technical changes.

\footnotetext{
${ }^{6}$ Respectively available at http://llvm.org/ and http://sourceforge.net/p/elftoolchain/wiki/Home/.
} 
tion 3. These tests are simply used as an indicator that the overhead introduced by the compiler is reasonable. More detailed measurements, detailing the strengths and limitations of particular PMA implementations are left for future work.

We have then taken a simple program and, using a hardware high-frequency timestamp, we have timed its performance in three cases, as presented in Fig. 13. The figure presents the average program execution time without any protection (in blue), with Fides (in red) and with Fides extended with the runtime checks provided by the security runtime (in beige).

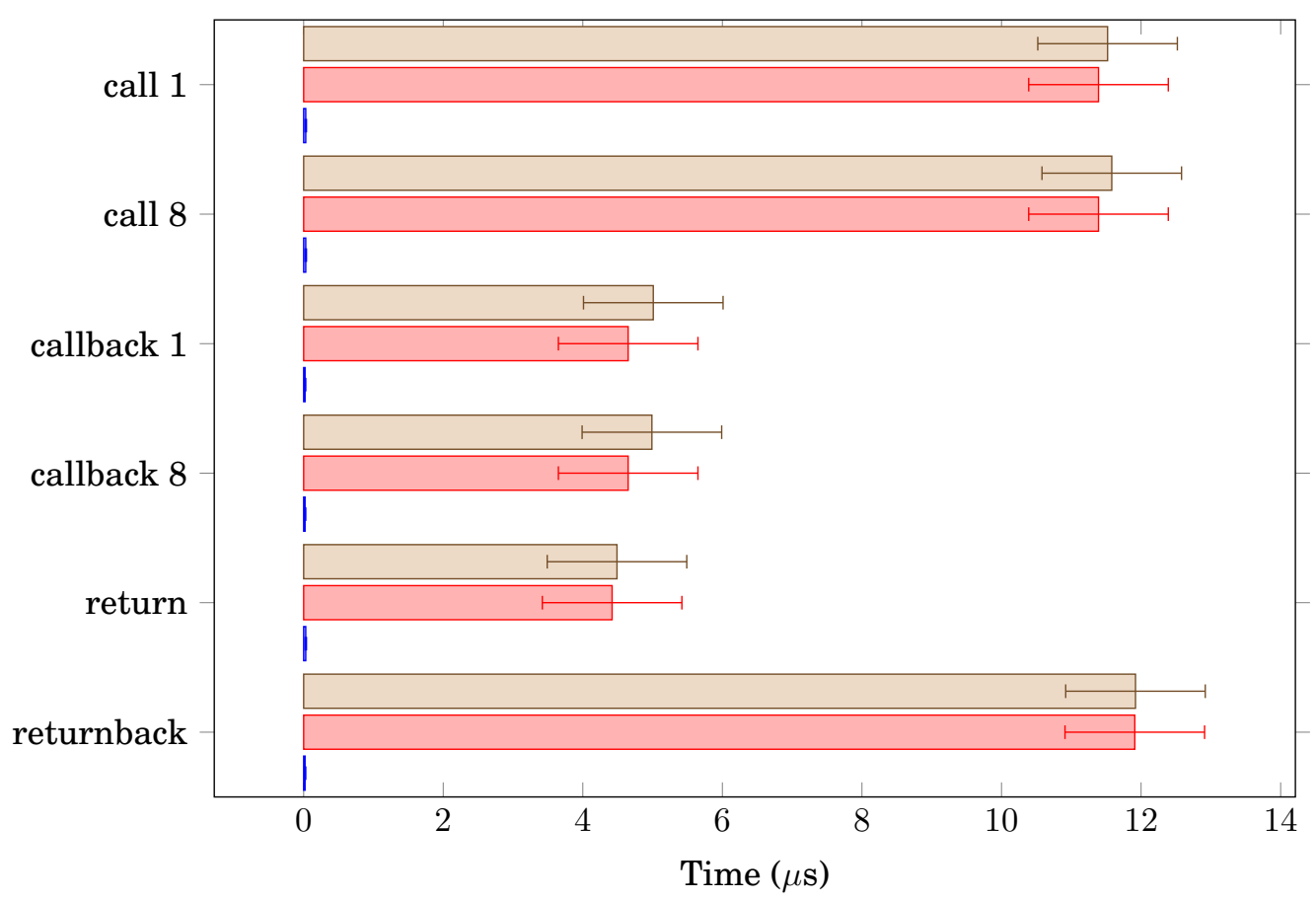

$\square$ Normal $^{\natural}$ Fides $^{\natural}$ Prototype

Fig. 13: Cost of different benchmarked instructions.

In Fig. 13, the y axis indicates which operations have been tested. The number following calls and callbacks indicates the number of arguments used and which trigger runtime checks. The security runtime adds checks to calls, callbacks, returns and returnbacks, so they are the only instructions that are considered. The "Normal" (blue) row indicates the cost of each operation without using the Fides architecture. The "Fides" (red) row indicates the cost of each operation while using the Fides architecture without the secure compilation scheme. The "Prototype" (beige) row the cost of each operation when the secure compilation scheme is used in addition to Fides. Each operation was performed 1000 times on a MacBook Pro with a $2.3 \mathrm{GHz}$ Intel Core i5 processor and 4GB 1333MHz DDR3 RAM. The difference between rows "Normal" and "Fides" shows the already high overhead of adopting the Fides architecture. The difference between rows "Fides" and "Prototype" is the overhead of the security checks introduced by the secure compiler, on average, this is a $\sim 3 \%$ overhead. Security checks are 
7.1.2. Secure Compilation of Cross-package Inheritance. Cross-package inheritance arises whenever a class from an export package extends a class from a different export package, as in Listing 12, where JointAccount from P-SUB extends Account from P-SUPER. Cross-package inheritance is not provided by $\mathcal{J}+\mathrm{E}$, as it breaks the encapsulation property of the language, creating a breach in the security of the language. Nevertheless, as there can be cases in which this feature is desirable, this paper now discusses how to securely implement it.

In order to allow cross-package inheritance and preserve as many benefits as possible from the encapsulation property of $\mathcal{J}+\mathrm{E}$, classes that can be extended must appear in import packages. Thus, given an import package, entry points are created not only for interface-defined methods, but also for class-defined ones and for constructors. Class JointAccount can optionally override methods of the super class Account, as is

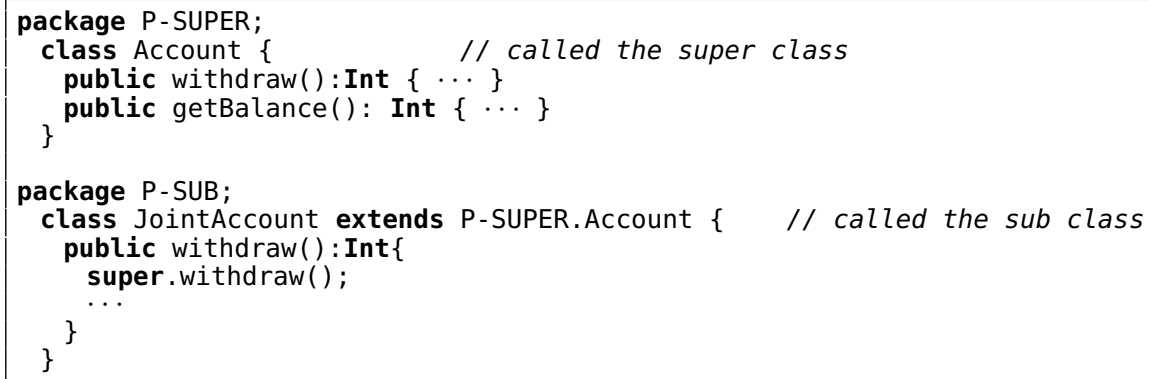

Listing 12: Example of cross-package inheritance.

the case with method withdraw(). Within those methods, calls to super can be used in order to call method withdraw () of the super class Account. Alternatively, if a method is not overridden (e.g. such as getBalance), calling $j$.getBalance () on an object $j$ of type JointAccount executes method getBalance() defined in the super class Account.

If the normal compilation scheme were followed, at the target-level $j$ is allocated to a single memory area where fields from classes Account and JointAccount are both allocated. If cross package inheritance involves only secure or only insecure classes, e.g. if P-SUPER and P-SUB from Listing 12 belong to the same component, it does not generate any problem. However, when secure classes can extend insecure ones and vice-versa, some complications arise, as presented in Problem 10.

Problem 10 (Allocation of $j$ ). Consider the case when Account is protected and JointAccount is not. If $j$ is allocated outside the protected memory partition, private fields of the Account subobject become accessible to external code. If $j$ is allocated inside the protected memory partition, two options arise. The first one is placing untrusted methods of JointAccount in the protected memory partition, violating the security of the compilation scheme. Otherwise, if methods of JointAccount are placed in the unprotected memory partition, they cannot access JointAccount's fields via offset. Getters and setters for fields of JointAccount could be exposed through entry points, but this would violate full abstraction, as those methods are not available at the high level.

These problems also arise when Account is not protected but JointAccount is, so compilation of cross-package inheritance cannot be achieved normally.

To overcome these difficulties, when $j$ is allocated, it is split in two sub-objects: $j a$, with fields of class Account, and $j j$, with fields of class JointAccount; the object identity of $j$ is $j j$ [van Dooren et al. 2013]. 
Consider firstly the case when Account is protected and JointAccount is not. External code needs to compile the expression $j=$ new JointAccount () so that it calls new Account ( ) to create object $j a$ in the protected memory section. External code must then save the resulting identifier for $j a$ to perform super calls, since they are translated as method calls. The additional checks inserted at entry points presented in Section 3 ensure that super calls are always well-typed.

Consider then the case when Account is not protected and JointAccount is. The secure compiler needs to call new JointAccount () and save the returned object identity for $j j$ in a memory location, since super calls in this case are compiled as callbacks. When expression $j=$ new JointAccount ( $)$ is compiled, the unprotected address $j a$ is stored at the low-level, right after the type of $j j$. The expression super.withdraw() is compiled as $j a$.withdraw().

The creation of two separate objects may seem to break full abstraction of the compilation scheme in a way similar to what Abadi found out for inner classes [Abadi 1999] in the early JVM. In fact, target level external code is given the functionality to call $j a$.withdraw(), which is not explicitly possible in the high-level language. However, $j$.super.withdraw() is an implicit call to the withdraw() method of Account, functionality that the high-level language already has. This way of handling cross-package inheritance does not add functionality at the target level, so it does not break full abstraction of the compilation scheme.

7.1.3. Secure Compilation of Inner Classes. Inner classes are classes that are defined inside another class, as in Listing 13 . Inner classes have access to private fields of the class they are defined within. They have not been included in the formalisation of $\mathcal{J}+\mathrm{E}$ as to keep it as simple as possible.

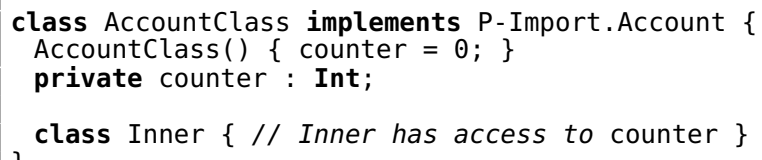

Listing 13: Example of an inner class.

Inner classes of the secure component are compiled as normal classes in the protected memory partition, in the usual fashion. To implement access from the inner class to the private fields of the surrounding class in a JVM style [Flanagan 1998], a getter and a setter for each private field are created. In the case of Listing 13, class AccountClass is extended with getters and setters for the counter field when compiled. Access from Inner to counter is compiled as method calls via the getter and setter.

This approach is inspired by Abadi [Abadi 1999], who shows that it breaks full abstraction of compilation in an early version of the JVM. In that setting, the additional low-level methods are not available at the high level, thus other low-level code besides the inner classes can call those methods, achieving something that was not possible at the high level. In our secure compilation scheme, the additional methods are available in the surrounding class. However the additional methods are not made available through entry points, thus the external code cannot invoke them. This means that the addition of inner classes to the secure compilation scheme preserves the full abstraction property. 
change the way references are counted and let parameters also increase the counter for an object reference. The return would decrease the counter for all parameters.

However, a second challenge arises: once a reference to an internal object is leaked to the unprotected code (such as for this in the callback above) the GC does not know when to deallocate such a reference. Here, an arguably safe methodology is to not deallocate a reference that is passed from the secure component to unprotected code. However, this creates problems when the allocated object is large or when many references are passed out.

An analogy that can be made now is that the secure GC faces challenges similar to those faced by distributed garbage collectors. Passing a reference to unprotected code is in fact analogous to passing a reference to a remote program: it is complex to know when such a reference can be deallocated. In the distributed setting this is due to communication problems and the impossibility for a garbage collector to inspect the object graph of a program on a remote machine. In this setting, this is due to the fact that the object graph in the unprotected memory section can be tampered with by the attacker. Unfortunately, in this setting the GC needs not only to be performant in case of interaction with unprotected code, it needs also to defend from potential attackers. Research on distributed garbage collection has developed several ways to addresses this problem [Abdullahi and Ringwood 1998], for example by giving each leaked reference a lease time. An unprotected object receiving a leased reference must periodically renew the lease on the reference, because once the lease time has expired, the reference is collected by the secure GC. This solution could be adopted in order to implement a secure memory manager, for it already addresses the need to provide a performant GC algorithm in case the unprotected code is well behaved. The details of the implementation, of how to make such a GC resilient against attacks and a more thorough treatment of the problems arising in various implementations are left for future work.

The proof that the garbage collector is secure remains a great challenge for the integration of secure compilation and GC. In any way such a proof is approached, GC notions (allocation, deallocation, references, the object graph etc) need to be carried into both the target and the source language in order to prove the compilation scheme between the two to be fully abstract. However, this causes the source-level programming model to become hindered by memory management - an additional way to let programmers introduce security flaws in their code. A way to overcome this challenge is to capture the behaviour of the GC in a different semantics of the source-level language, an extended semantics. Full abstraction of the compilation scheme should then be proven with respect to the extended semantics. This treatment would not clutter the source language with explicit GC, but it would still capture the effect of GC at the source level. The extended semantics should capture the behaviour of the GC as it is at the target level, so that problems as those highlighted above do not arise, since they are captured at the source level as well. Additionally, the extended semantics should be proven to be secure w.r.t the normal operational semantics: this would guarantee that the behaviour of the GC does not introduce security leaks.

Devising such an extended semantics and proving (i) that it is not introducing security leaks and (ii) a fully abstract translation involving it are left for future work.

\section{RELATED WORK}

Secure compilation through full abstraction was pioneered by Abadi [Abadi 1999], where, alongside a result in the $\pi$-calculus setting, Java bytecode compilation in the early JVM is shown to expose methods used to access private fields by private inner classes. Kennedy [Kennedy 2006] listed six full abstraction failures in the compilation to .NET, half of which have been fixed in modern $C^{\#}$ implementations. 
Address space layout randomisation has been adopted by Abadi and Plotkin [Abadi and Plotkin 2012] and subsequently by Jagadeesan et al. [Jagadeesan et al. 2011] to guarantee probabilistic full abstraction of a compilation scheme. In both works the lowlevel language is more high-level than ours and the protection mechanism is different. Compilation does not necessarily need to target machine code, as Fournet et al. [Fournet et al. 2013] show by providing a fully abstract compilation scheme from an ML dialect named $\mathrm{F}^{*}$ to JavaScript that relies on type-based invariants. Similarly, Ahmed and Blume [Ahmed and Blume 2011] prove full abstraction of a continuation-passing style translation from simply-typed $\lambda$-calculus to System F. In both works, the targetlevel language is typed and more high-level than the one of this paper. The checks introduced by our compilation scheme are simpler than the checks of Fournet et al., so secure compilation seems easier to implement for object-oriented languages than for functional ones. An alternative target language for secure compilation is Typed Assembly Language (TAL), which was proposed in the work of Morrisett et al. [Morrisett et al. 1999]. The presence of the Intel SGX processor strengthens our choice of the PMA architecture for the target language over TAL.

Adopting a fine-grained, program counter-based memory access control mechanism to achieve secure compilation was pioneered by Agten et al. [Agten et al. 2012] and then investigated by Patrignani et al. [Patrignani et al. 2013]. This paper wraps both results in a coherent presentation, extending certain aspects of both papers.

A large amount of work on secure compilation involved the compilation of unsafe languages such as C. An extensive survey can be found in Younan et al. [Younan et al. 2012]. Instead of focussing on a fully abstract compilation, that research is devoted to strengthening the security properties of $\mathrm{C}$.

Correct (and certified) compilation, are very broad research fields that aim at providing compilers that preserve contextual equivalence between the source and target language [Chlipala 2007; Leroy 2009]. The main difference between those fields and secure compilation is the nature of low-level contexts. In order to model a powerful lowlevel attacker, we have considered an arbitrary complex low-level context interacting with the compiled components. In those works, the low-level context is assumed to be obtained through compilation, so it does not misbehave. A great contribution of correct compilation works is that they provide compilers for which Assumption 1 holds.

Different security architectures with access control mechanisms comparable to ours have been developed in the last years: TrustVisor [McCune et al. 2010], Flicker [McCune et al. 2008], Nizza [Singaravelu et al. 2006] SPMs [Strackx et al. 2010; Strackx and Piessens 2012] and the Intel SGX [McKeen et al. 2013]. Similar architectures with fine-grained protection schemes have also been employed to develop less monolithic operating systems [Witchel et al. 2002]. A common characteristic of these security architectures is that they rely on a small TCB, either only the hardware, or the hardware plus a hypervisor small enough to be formally verifiable [Vasudevan et al. 2013]. The existence of industry prototypes alongside research ones underlines the feasibility bringing efficient and secure low-level memory access control in commodity hardware. No results comparable to ours have been proven for these systems.

\section{CONCLUSION AND FUTURE WORK}

This paper presented a fully abstract compilation scheme for a strongly-typed, singlethreaded, component-based, object-oriented programming language with dynamic memory allocation and exceptions. The compilation scheme targets untyped assembly code enhanced with a protected modules architecture. From the security perspective a fully abstract compilation scheme ensures that target-level attackers are restricted to the same powers source-level attackers have. Additionally, it guarantees source-level reasoning: in order to understand how a program behaves, it is sufficient to inspect its 
source code. This paper highlighted mistakes that make a naïve compilation scheme not fully abstract and how to correct them. The compilation scheme is proven to be fully abstract, guaranteeing preservation and reflection of contextual equivalence between high-level components and their compiled counterparts.

As presented in Section 7, integrating advanced language features such as garbage collection with securely compiled code is a challenging and relevant research direction. Additionally, providing secure compilers for multicore architectures and for multiprincipal scenarios (i.e. involving more than one protected partition, each with code coming from different stakeholders) are important, future work challenges. Finally, the efficient transfer of large data values will impact on the performance and usability of implementations of secure compilers, this is also a challenge that is left for future work.

Acknowledgements We would like to thanks the anonymous reviewers for their insightful comments and suggestions on previous versions of this paper.

\section{References}

Martín Abadi. 1999. Protection in programming-language translations. In Secure Internet programming. Springer-Verlag, London, UK, 19-34. http://dl.acm.org/citation.cfm?id=380171.380174

Martín Abadi and Gordon D. Plotkin. 2012. On Protection by Layout Randomization. ACM Trans. Inf. Syst. Secur. 15, 2, Article 8 (July 2012), 29 pages. D0I : http://dx.doi.org/10.1145/2240276.2240279

Saleh E. Abdullahi and Graem A. Ringwood. 1998. Garbage Collecting the Internet: A Survey of Distributed Garbage Collection. ACM Comput. Surv. 30, 3 (Sept. 1998), 330-373. DOI : http://dx.doi.org/10.1145/292469.292471

Pieter Agten, Bart Jacobs, and Frank Piessens. 2015. Sound Modular Verification of C Code Executing in an Unverified Context. In Proceedings of the 42nd Annual ACM SIGPLAN-SIGACT Symposium on Principles of Programming Languages (POPL '15).

Pieter Agten, Raoul Strackx, Bart Jacobs, and Frank Piessens. 2012. Secure Compilation to Modern Processors. In Proceedings of the 2012 IEEE 25th Computer Security Foundations Symposium (CSF '12). IEEE Computer Society, Washington, DC, USA, Article 12, 15 pages. DOI : http://dx.doi.org/10.1109/CSF.2012.12

Amal Ahmed and Matthias Blume. 2011. An equivalence-preserving CPS translation via multi-language semantics. SIGPLAN Not. 46, 9, Article 30 (Sept. 2011), 14 pages. DOI : http://dx.doi.org/10.1145/2034574.2034830

Ittai Anati, Shay Gueron, S Johnson, and V Scarlata. 2013. Innovative Technology for CPU Based Attestation and Sealing. In Proceedings of the 2nd International Workshop on Hardware and Architectural Support for Security and Privacy, HASP, Vol. 13. http://software.intel.com/sites/default/files/article/413939/ hasp-2013-innovative-technology-for-attestation-and-sealing.pdf

Niels Avonds, Raoul Strackx, Pieter Agten, and Frank Piessens. 2013. Salus: Non-Hierarchical Memory Access Rights to Enforce the Principle of Least Privilege. In Security and Privacy in Communication Networks (SecureComm'13).

Adam Chlipala. 2007. A certified type-preserving compiler from lambda calculus to assembly language. SIGPLAN Not. 42, 6, Article 5 (June 2007), 12 pages. D0I : http://dx.doi.org/10.1145/1273442.1250742

Pierre-Louis Curien. 2007. Definability and Full Abstraction. Electron. Notes Theor. Comput. Sci. 172 (April 2007), 301-310. D0I : http://dx.doi.org/10.1016/j.entcs.2007.02.011

Frank S. de Boer, Marcello M. Bonsangue, Martin Steffen, and Erika Ábrahám. 2005. A fully abstract semantics for UML components. In Proceedings of the Third international conference on Formal Methods for Components and Objects (FMCO'04). Springer-Verlag, Berlin, Heidelberg, 49-69. D0I : http://dx.doi.org/10.1007/11561163_3

Roland Ducournau. 2011. Implementing statically typed object-oriented programming languages. $A C M$ Comput. Surv. 43, 3, Article 18 (April 2011), 48 pages. D0I : http://dx.doi.org/10.1145/1922649.1922655

Karim Eldefrawy, Aurélien Francillon, Daniele Perito, and Gene Tsudik. 2012. SMART: Secure and Minimal Architecture for (Establishing a Dynamic) Root of Trust. In NDSS 2012, 19th Annual Network and Distributed System Security Symposium. San Diego, United States.

Ulfar Erlingsson, Yves Younan, and Frank Piessens. 2010. Low-level software security by example. In Handbook of Information and Communication Security. Springer, Berlin, Heidelberg, 663-658. https: //irias.kuleuven.be/handle/123456789/267049 
David Flanagan. 1998. Java in a Nutshell. Deutsche Ausgabe der 2. A. O’Reilly.

Cedric Fournet, Nikhil Swamy, Juan Chen, Pierre-Evariste Dagand, Pierre-Yves Strub, and Benjamin Livshits. 2013. Fully abstract compilation to JavaScript. SIGPLAN Not. 48, 1, Article 26 (Jan. 2013), 14 pages. DOI : http://dx.doi.org/10.1145/2480359.2429114

J.A. Halderman, S.D. Schoen, N. Heninger, W. Clarkson, W. Paul, J.A. Calandrino, A.J. Feldman, J. Appelbaum, and E.W. Felten. 2008. Lest we remember: Cold boot attacks on encryption keys. In USENIX Security Symposium. 45-60. http://www.cs.umass.edu/ ransford/srg/papers/halderman--coldboot.pdf

Matthew Hoekstra, Reshma Lal, Pradeep Pappachan, Vinay Phegade, and Juan Del Cuvillo. 2013. Using innovative instructions to create trustworthy software solutions. In Proceedings of the 2nd International Workshop on Hardware and Architectural Support for Security and Privacy. ACM, 11. http://software. intel.com/sites/default/files/article/413938/hasp-2013-innovative-instructions-for-trusted-solutions.pdf

Andrei Homescu, Steven Neisius, Per Larsen, Stefan Brunthaler, and Michael Franz. 2013. Profile-guided Automated Software Diversity. In Proceedings of the 2013 IEEE/ACM International Symposium on Code Generation and Optimization (CGO) (CGO '13). IEEE Computer Society, Washington, DC, USA, 1-11. DOI : http://dx.doi.org/10.1109/CGO.2013.6494997

Intel Corporation. 2013. Software Guard Extensions Programming Reference. http://software.intel.com/sites/ default/files/329298-001.pdf

Radha Jagadeesan, Corin Pitcher, Julian Rathke, and James Riely. 2011. Local Memory via Layout Randomization. In Proceedings of the 2011 IEEE 24th Computer Security Foundations Symposium (CSF '11). IEEE Computer Society, Washington, DC, USA, 161-174. DOI : http://dx.doi.org/10.1109/CSF.2011.18

Alan Jeffrey and Julian Rathke. 2005a. A fully abstract may testing semantics for concurrent objects. Theor. Comput. Sci. 338, 1-3 (June 2005), 17-63. D0I : http://dx.doi.org/10.1016/j.tcs.2004.10.012

Alan Jeffrey and Julian Rathke. 2005b. Java Jr.: fully abstract trace semantics for a core Java language. In ESOP'05 (LNCS), Vol. 3444. Springer, 423-438. D0I : http://dx.doi.org/10.1007/978-3-540-31987-0_29

Andrew Kennedy. 2006. Securing the .NET programming model. Theor. Comput. Sci. 364, 3 (Nov. 2006), 311-317. DOI : http://dx.doi.org/10.1016/j.tcs.2006.08.014

Per Larsen, Stefan Brunthaler, and Michael Franz. 2014. Security through Diversity: Are We There Yet? IEEE Security \& Privacy 12, 2 (2014), 28-35. D0I : http://dx.doi.org/10.1109/MSP.2013.129

Chris Lattner and Vikram Adve. 2004. LLVM: A Compilation Framework for Lifelong Program Analysis \& Transformation. In Proceedings of the 2004 International Symposium on Code Generation and Optimization (CGO'04). Palo Alto, California.

Xavier Leroy. 2009. A Formally Verified Compiler Back-end. J. Autom. Reason. 43, 4 (Dec. 2009), 363-446. DOI : http://dx.doi.org/10.1007/s10817-009-9155-4

Jonathan M. McCune, Yanlin Li, Ning Qu, Zongwei Zhou, Anupam Datta, Virgil Gligor, and Adrian Perrig. 2010. TrustVisor: Efficient TCB Reduction and Attestation. In SP '10. IEEE, Washington, DC, USA, 143-158. DOI : http://dx.doi.org/10.1109/SP.2010.17

Jonathan M. McCune, Bryan J. Parno, Adrian Perrig, Michael K. Reiter, and Hiroshi Isozaki. 2008. Flicker: an execution infrastructure for TCB minimization. SIGOPS Oper. Syst. Rev. 42, 4, Article 24 (April 2008), 14 pages. DOI : http://dx.doi.org/10.1145/1357010.1352625

Frank McKeen, Ilya Alexandrovich, Alex Berenzon, Carlos V. Rozas, Hisham Shafi, Vedvyas Shanbhogue, and Uday R. Savagaonkar. 2013. Innovative instructions and software model for isolated execution. In HASP '13. ACM, Article 10, 1 pages. D0I : http://dx.doi.org/10.1145/2487726.2488368

Greg Morrisett, David Walker, Karl Crary, and Neal Glew. 1999. From System F to Typed Assembly Language. ACM Trans. Program. Lang. Syst. 21, 3 (May 1999), 527-568. D0I : http://dx.doi.org/10.1145/319301.319345

Job Noorman, Pieter Agten, Wilfried Daniels, Raoul Strackx, Anthony Van Herrewege, Christophe Huygens, Bart Preneel, Ingrid Verbauwhede, and Frank Piessens. 2013. Sancus: Low-cost trustworthy extensible networked devices with a zero-software Trusted Computing Base. In Proceedings of the 22nd USENIX conference on Security symposium. USENIX Association.

Marco Patrignani and Dave Clarke. 2014. Fully Abstract Trace Semantics of Low-level Isolation Mechanisms. In Proceedings of the 29th Annual ACM Symposium on Applied Computing (SAC 2014).

Marco Patrignani, Dave Clarke, and Frank Piessens. 2013. Secure Compilation of Object-Oriented Components to Protected Module Architectures. In (APLAS'13) (LNCS), Vol. 8301. 176-191.

Gordon D. Plotkin. 1977. LCF Considered as a Programming Language. Theoretical Computer Science 5 (1977), 223-255.

Ryan Roemer, Erik Buchanan, Hovav Shacham, and Stefan Savage. 2012. Return-Oriented Programming: Systems, Languages, and Applications. ACM Trans. Inf. Syst. Secur. 15, 1, Article 2 (March 2012), 34 pages. DOI : http://dx.doi.org/10.1145/2133375.2133377 
Hovav Shacham, Matthew Page, Ben Pfaff, Eu-Jin Goh, Nagendra Modadugu, and Dan Boneh. 2004. On the Effectiveness of Address-space Randomization. In Proceedings of the 11th ACM Conference on Computer and Communications Security (CCS '04). ACM, New York, NY, USA, 298-307. DOI : http://dx.doi.org/10.1145/1030083.1030124

Lenin Singaravelu, Calton Pu, Hermann Härtig, and Christian Helmuth. 2006. Reducing TCB complexity for security-sensitive applications: three case studies. SIGOPS Oper. Syst. Rev. 40, 4, Article 13 (April 2006), 14 pages. DOI : http://dx.doi.org/10.1145/1218063.1217951

Raoul Strackx, Job Noorman, Ingrid Verbauwhede, Bart Preneel, and Frank Piessens. 2013. Protected Software Module Architectures. In ISSE 2013 Securing Electronic Business Processes, Helmut Reimer, Norbert Pohlmann, and Wolfgang Schneider (Eds.). Springer Fachmedien Wiesbaden, 241-251. DOI : http://dx.doi.org/10.1007/978-3-658-03371-2_21

Raoul Strackx and Frank Piessens. 2012. Fides: selectively hardening software application components against kernel-level or process-level malware. In Proceedings of the 2012 ACM conference on Computer and communications security (CCS '12). ACM, New York, NY, USA, 2-13. DOI : http://dx.doi.org/10.1145/2382196.2382200

Raoul Strackx, Frank Piessens, and Bart Preneel. 2010. Efficient Isolation of Trusted Subsystems in Embedded Systems. In SecureComm. 344-361.

Raoul Strackx, Yves Younan, Pieter Philippaerts, Frank Piessens, Sven Lachmund, and Thomas Walter. 2009. Breaking the memory secrecy assumption. In Proceedings of the Second European Workshop on System Security (EUROSEC '09). ACM, New York, NY, USA, Article 1, 8 pages. DOI : http://dx.doi.org/10.1145/1519144.1519145

Marko van Dooren, Dave Clarke, and Bart Jacobs. 2013. Subobject-Oriented Programming. In Formal Methods for Components and Objects (Lecture Notes in Computer Science), Vol. 7866. Springer Berlin Heidelberg, 38-82. D0I : http://dx.doi.org/10.1007/978-3-642-40615-7_2

Amit Vasudevan, Sagar Chaki, Limin Jia, Jonathan McCune, James Newsome, and Anupam Datta. 2013. Design, Implementation and Verification of an eXtensible and Modular Hypervisor Framework. In Proceedings of the 2013 IEEE Symposium on Security and Privacy (SP '13). IEEE Computer Society, Washington, DC, USA, 430-444. D0I: http://dx.doi.org/10.1109/SP.2013.36

Richard Wartell, Vishwath Mohan, Kevin W. Hamlen, and Zhiqiang Lin. 2012. Binary Stirring: Selfrandomizing Instruction Addresses of Legacy x86 Binary Code. In Proceedings of the 2012 ACM Conference on Computer and Communications Security (CCS '12). ACM, New York, NY, USA, 157-168. DOI : http://dx.doi.org/10.1145/2382196.2382216

Johannes Winter and Kurt Dietrich. 2012. A Hijacker's Guide to the LPC Bus, In Proceedings of the 8th European conference on Public Key Infrastructures, Services, and Applications (EuroPKI'11). Public Key Infrastructures, Services and Applications (2012), 176-193.

Emmett Witchel, Josh Cates, and Krste Asanović. 2002. Mondrian Memory Protection. SIGPLAN Not. 37, 10 (Oct. 2002), 304-316. D0I : http://dx.doi.org/10.1145/605432.605429

Yves Younan. 2008. Efficient Countermeasures for Software Vulnerabilities due to Memory Management Errors. Ph.D. Dissertation. Informatics Section, Department of Computer Science, Faculty of Engineering Science. https://irias.kuleuven.be/handle/1979/1765 Joosen, Wouter and Piessens, Frank (supervisors).

Yves Younan, Wouter Joosen, and Frank Piessens. 2012. Runtime countermeasures for code injection attacks against C and C++ programs. Comput. Surveys 44, 3 (2012), 17:1-17:28. https://irias.kuleuven. be/handle/123456789/288462

Received ; revised ; accepted 